\title{
Critical endpoint of the finite temperature phase transition for three flavor QCD
}

\author{
Xiao-Yong Jin, ${ }^{1, \dagger}$ Yoshinobu Kuramashi, ${ }^{2,3,1}$ Yoshifumi Nakamura, ${ }^{1,4, *}$ Shinji Takeda, ${ }^{5,1}$ and Akira Ukawa \\ ${ }^{1}$ RIKEN Advanced Institute for Computational Science, Kobe, Hyogo 650-0047, Japan \\ ${ }^{2}$ Faculty of Pure and Applied Sciences, University of Tsukuba, Tsukuba, Ibaraki 305-8571, Japan \\ ${ }^{3}$ Center for Computational Sciences, University of Tsukuba, Tsukuba, Ibaraki 305-8577, Japan \\ ${ }^{4}$ Department of Computational Sciences, Graduate School of System Informatics, Kobe University, Kobe, \\ Hyogo 657-8501, Japan \\ ${ }^{5}$ Institute of Physics, Kanazawa University, Kanazawa 920-1192, Japan
}

(Received 24 November 2014; published 26 January 2015)

\begin{abstract}
We investigate the critical endpoint of finite temperature phase transition of $N_{f}=3$ QCD at zero chemical potential. We employ the renormalization-group improved Iwasaki gauge action and nonperturbatively $O(a)$-improved Wilson-clover fermion action. The critical endpoint is determined by using the intersection point of kurtosis for the temporal size $N_{t}=4,6$, 8. Spatial sizes of $N_{l}=6-16\left(N_{t}=4\right)$, 10-24 $\left(N_{t}=6\right)$, and 12-24 $\left(N_{t}=8\right)$ are employed. We find that $N_{t}=4$ is out of the scaling region. Using results for $N_{t}=6$ and 8 and making linear extrapolations in $1 / N_{t}^{2}$, we obtain $\sqrt{t_{0}} T_{\mathrm{E}}=0.0988(14)(9)$, $\sqrt{t_{0}} m_{\mathrm{PS}, \mathrm{E}}=0.2274(52)(108)$, and $m_{\mathrm{PS}, \mathrm{E}} / T_{\mathrm{E}}=2.302(62)(13)$, where the first error is statistical error, the second error is systematic error, and $m_{\mathrm{PS}}$ is the pseudoscalar meson mass. If one uses $1 / \sqrt{t_{0}}=1.347(30) \mathrm{GeV}$, as reported by Borsanyi et al., one finds $T_{\mathrm{E}}=133(2)(1)(3) \mathrm{MeV}$, $m_{\mathrm{PS}, \mathrm{E}}=306(7)(14)(7) \mathrm{MeV}$, and $m_{\mathrm{PS}, \mathrm{E}} / m_{\mathrm{PS}}^{\mathrm{phys}, \mathrm{sym}}=0.745(17)(35)(17)$, where the third error comes from the error of $\sqrt{t_{0}}$ and $m_{\mathrm{PS}}^{\mathrm{phys}, \mathrm{sym}}=\sqrt{\left(m_{\pi}^{2}+2 m_{K}^{2}\right) / 3}$. Our current estimation of $\sqrt{t_{0}} m_{\mathrm{PS}, \mathrm{E}}$ in the continuum limit is about $25 \%$ smaller than the $\mathrm{SU}(3)$ symmetric point.
\end{abstract}

DOI: 10.1103/PhysRevD.91.014508

PACS numbers: $11.15 . \mathrm{Ha}, 12.38 . \mathrm{Gc}, 25.75 . \mathrm{Nq}$

\section{INTRODUCTION}

Knowledge of QCD phase structure is the basis for understanding the physics of the strong interactions at finite temperature and density. Since simulations with finite chemical potential are plagued with the sign problem, it is important to clearly understand the finite temperature phase diagram as a function of light up-down quark masses and a strange quark mass before starting extensive studies at finite chemical potential.

Analytical arguments indicate that the finite temperature transition with three massless quarks is of first order [1], which should then extend into the region of finite quark masses, ending at a line of critical points belonging to the $\mathrm{Z}(2)$ universality class [2]. Theoretical considerations alone cannot tell if the physical point with $m_{u d} / m_{s} \approx 25$ lies on the crossover side or the first order side of the critical line.

The results of simulations done to date to locate the critical line are rather confusing. All results with staggered fermion action are consistent with the physical point being in the crossover region [3-8]. However, on the location of the critical line, while the standard staggered action yields the critical quark mass as large as the physical up-down quark masses for both $N_{t}=4$ [3-6] and $N_{t}=6$ [5], more

\footnotetext{
*nakamura@ riken.jp

${ }^{\dagger}$ Present address: Argonne Leadership Computing Facility, Argonne National Laboratory, Argonne, Illinois 60439, USA.
}

recent studies with improved staggered actions indicate that the critical quark mass is significantly smaller than the physical up-down quark masses $[7,8]$. In fact, improved staggered actions have not yet found first order signals. On the other hand, with the Wilson quark action, an extensive pioneering study with naive Wilson action and $N_{t}=4$ temporal lattice size found that the physical point lies in the first order phase transition region [9].

To clarify the issue, we study the critical endpoint for $N_{f}=3$ lattice QCD with nonperturbatively $O(a)$ improved Wilson fermion action, and we attempt to estimate the continuum limit. Wilson-type fermion action has exact flavor symmetry. In contrast, taste breaking with staggered-type fermion action becomes large at coarse lattice spacings [10]. We consider this to be a potentially more serious issue in comparison to lack of chiral symmetry with Wilson-type fermion actions. Indeed, the lack of chiral symmetry should not be a major problem for studying the critical endpoint which is expected to take place at a finite value of the quark mass.

This paper is organized as follows. In Sec. II we recapitulate the finite size scaling of kurtosis which we employ to locate the critical endpoint. In Sec. III we describe our observables, taking chiral condensate as an example. In Sec. IV we present the simulation details, including the parameters and the simulation algorithm. Our numerical results are presented in Sec. V. Our conclusions are summarized in Sec. VI. 


\section{METHOD}

Consider an observable $\mathcal{O}$. If one denotes by $x$ the conjugate variable to $\mathcal{O}$, the $n$th cumulant $\kappa_{n}$ of $\mathcal{O}$ is defined by

$$
\log \langle\exp (x \mathcal{O})\rangle=\sum_{n=1}^{\infty} \frac{x^{n}}{n !} \kappa_{n}
$$

The mean value $O$ of $\mathcal{O}$, susceptibility $\chi_{\mathcal{O}}$, skewness $S_{\mathcal{O}}$, and kurtosis $K_{\mathcal{O}}$ are defined as

$$
\begin{gathered}
O=\kappa_{1} / V=\langle\mathcal{O}\rangle / V, \\
\chi_{\mathcal{O}}=\kappa_{2} / V=\left\langle(\mathcal{O}-\langle\mathcal{O}\rangle)^{2}\right\rangle / V, \\
S_{\mathcal{O}}=\frac{\kappa_{3}}{\kappa_{2}^{3 / 2}}, \\
K_{\mathcal{O}}=\frac{\kappa_{4}}{\kappa_{2}^{2}},
\end{gathered}
$$

where $V$ is volume. Of particular interest for locating the critical endpoint is kurtosis $K_{\mathcal{O}}$ since it is expected to have no or only a small finite size effect at a second order transition point.

Let $F(t, h, L)$ be the free energy of a system with a second order phase transition at $t=h=0$ and a linear extent $L$. The two parameters $t$ and $h$ are reduced "temperature" and "external field" variables conjugate to the "energy" $\mathcal{E}$ and "magnetization" $\mathcal{M}$ operator characterizing the renormalization group flow around the critical point. According to finite size scaling theory, the free energy scales as $F(t, h, L)=F\left(t L^{y_{t}}, h L^{y_{h}}, 1\right)$ up to analytic terms. If one approaches the critical point along the line $h=0$, the kurtosis for the magnetization $\mathcal{M}$ satisfies the scaling relation

$$
K_{\mathcal{M}}=\frac{F^{(4)}\left(t L^{y_{t}}\right)}{F^{(2)}\left(t L^{y_{t}}\right)^{2}}
$$

with $F^{(n)}(t)=\partial^{n} F(t, h, 1) /\left.\partial h^{n}\right|_{h=0}$. Hence the kurtosis is expected to have a fixed value at $t=0$ independent of the size $L$.

One can generalize the scaling relation to a more general class of operators $\mathcal{O}$ which may be expressed as a linear combination of $\mathcal{E}$ and $\mathcal{M}$ close to the second order transition point. If the magnetization exponent $y_{h}$ is larger than the thermal exponent $y_{t}$, one finds

$K_{\mathcal{O}}=\mathcal{F}_{\mathcal{O}}\left(t L^{y_{t}}\right)+L^{y_{t}-y_{h}} \mathcal{F}^{\prime}{ }_{\mathcal{O}}\left(t L^{y_{t}}\right)+O\left(L^{2\left(y_{t}-y_{h}\right)}\right)$,

where $\mathcal{F}(x)$ is the scaling function for the leading term and $\mathcal{F}^{\prime}(x)$ is the subleading term, which is smaller by a factor
$L^{y_{t}-y_{h}}$. Thus there is a size-dependent shift of kurtosis in the general case.

At the first order phase transition point, for large volumes, kurtosis reaches the minimum [11] according to

$$
K_{\mathcal{O}}=-2+\frac{c}{N_{l}^{d}}+O\left(1 / N_{l}^{2 d}\right)
$$

where $d$ is the dimension.

We use the property of $K$ discussed above to determine the critical endpoint by the following strategy.

(1) For a given temporal lattice size $N_{t}$ and a spatial size $N_{l}$, we collect data for a set of values of inverse gauge coupling $\beta$ and hopping parameter $\kappa$ around the critical endpoint.

(2) For each $N_{t}$ and $N_{l}$, we determine the transition point from the peak of susceptibility; this is done either by fixing $\beta$ and fitting the susceptibility as a function of $\kappa$ or by fixing $\kappa$ and searching the peak by reweighting in $\beta$ [12].

(3) Using the value of $\kappa$ at the peak, we determine the kurtosis at the peak $K_{\mathrm{t}}$ for each spatial lattice size $N_{l}$ as a function of $\beta$.

(4) Plotting $K_{\mathrm{t}}$ as a function of $\beta$ for a set of spatial sizes $N_{l}$, we determine the critical endpoint by estimating the point where the kurtosis at different $N_{l}$ intersects; this is done by a fit inspired by the leading term of finite size scaling [5]:

$$
K=K_{\mathrm{E}}+a N_{l}^{1 / \nu}\left(\beta-\beta_{\mathrm{E}}\right),
$$

where $K_{\mathrm{E}}$ and $\beta_{\mathrm{E}}$ are the values of $K$ and $\beta$ at the critical endpoint, respectively. In Fig. 1 we sketch our method.

It is expected that the critical endpoint of $N_{f}=3$ (also $N_{f}=2+1$ ) QCD belongs to the universality class of the 3D Ising model with Z(2) symmetry [2]. Therefore the values $K_{\mathrm{E}}=-1.396$ and $\nu=0.630$ are expected. However, we do not assume any values for $K_{\mathrm{E}}$ and $\nu$ in the fitting.

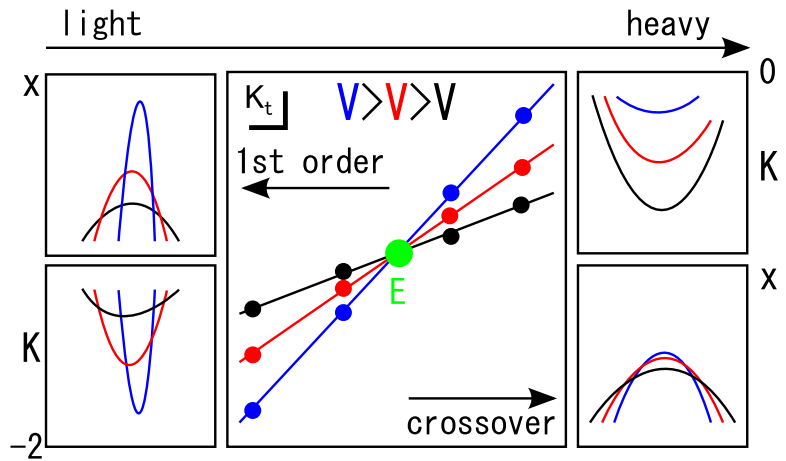

FIG. 1 (color online). Schematic picture of a method to determine the critical endpoint. 


\section{OBSERVABLES}

We can write the partition function with Wilson-clover fermion action as

$$
Z=\int d U e^{-S_{g}} \prod_{i=1}^{N_{f}} \operatorname{det} D_{i},
$$

where

$$
\begin{aligned}
D_{i} & =\frac{1}{2 \kappa_{i}}+C-\frac{1}{2} \sum_{\mu=1}^{4}\left(T_{\mu}^{+}+T_{\mu}^{-}\right), \\
C & =\frac{i}{4} c_{\mathrm{SW}} \sigma_{\mu \nu} F_{\mu \nu}(n) \delta_{m, n}, \\
T_{\mu}^{ \pm} & =\left(1 \mp \gamma_{\mu}\right) U_{ \pm \mu}(n) \delta_{n, m \pm \hat{\mu}}, \\
\operatorname{am}_{i} & =\frac{1}{2 \kappa_{i}}+\text { const. }
\end{aligned}
$$

Let us introduce the derivatives,

$$
\begin{aligned}
W_{1}\left(m_{i}\right)= & \frac{\partial \log \operatorname{det} D_{i}\left(m_{i}\right)}{\partial a m_{i}}=\operatorname{Tr}\left[D_{i}^{-1}\right], \\
W_{2}\left(m_{i}\right)= & \frac{\partial W_{1}\left(m_{i}\right)}{\partial a m_{i}}=-\operatorname{Tr}\left[D_{i}^{-2}\right], \\
W_{3}\left(m_{i}\right)= & \frac{\partial^{2} W_{1}\left(m_{i}\right)}{\partial\left(a m_{i}\right)^{2}}=2 \operatorname{Tr}\left[D_{i}^{-3}\right], \\
W_{4}\left(m_{i}\right)= & \frac{\partial^{3} W_{1}\left(m_{i}\right)}{\partial\left(a m_{i}\right)^{3}}=-6 \operatorname{Tr}\left[D_{i}^{-4}\right], \\
W_{j}\left(m_{i}\right)= & \frac{\partial^{j-1} W_{1}\left(m_{i}\right)}{\partial\left(a m_{i}\right)^{j-1}}=(-1)^{j-1} \times(j-1) ! \\
& \times \operatorname{Tr}\left[D_{i}^{-j}\right],
\end{aligned}
$$

and the flavor averages,

$$
\bar{W}_{j}=\frac{1}{N_{f}} \sum_{i}^{N_{f}} W_{j}\left(m_{i}\right) .
$$

We consider derivatives of $X=\prod_{i}^{N_{f}} \operatorname{det} D_{i}$ with respect to $a m_{i}$ up to fourth order. Defining

$$
\frac{\partial^{j}}{\partial(a m)^{j}}=\left(\frac{1}{N_{f}} \sum_{i}^{N_{f}} \frac{\partial}{\partial a m_{i}}\right)^{j},
$$

we find $\frac{\partial}{\partial(a m)} X=X \bar{W}_{1} \equiv X Q_{1}$,

$\frac{\partial^{2}}{\partial(a m)^{2}} X=X\left[\bar{W}_{2}+\bar{W}_{1}^{2}\right] \equiv X Q_{2}$,

$\frac{\partial^{3}}{\partial(a m)^{3}} X=X\left[\bar{W}_{3}+3 \bar{W}_{2} \bar{W}_{1}+\bar{W}_{1}^{3}\right] \equiv X Q_{3}$,

$\frac{\partial^{4}}{\partial(a m)^{4}} X=X\left[\bar{W}_{4}+4 \bar{W}_{3} \bar{W}_{1}+3 \bar{W}_{2}^{2}+6 \bar{W}_{2} \bar{W}_{1}^{2}+\bar{W}_{1}^{4}\right]$

$$
\equiv X Q_{4}
$$

We find then that the mean value $\Sigma$ and the susceptibility $\chi_{\Sigma}$ are given by

$$
\begin{aligned}
\Sigma & \equiv \frac{1}{N_{l}^{3} N_{t}} \frac{\partial \ln Z}{\partial a m}=\frac{\left\langle Q_{1}\right\rangle}{N_{l}^{3} N_{t}}, \\
\chi_{\Sigma} & \equiv \frac{1}{N_{l}^{3} N_{t}} \frac{\partial^{2} \ln Z}{\partial(a m)^{2}}=\frac{\left\langle Q_{2}\right\rangle-\left\langle Q_{1}\right\rangle^{2}}{N_{l}^{3} N_{t}},
\end{aligned}
$$

and skewness $S_{\Sigma}$ and kurtosis $K_{\Sigma}$ by

$$
\begin{aligned}
S_{\Sigma} & =\frac{\partial^{3} \ln Z}{\partial(a m)^{3}} /\left(\frac{\partial^{2} \ln Z}{\partial(a m)^{2}}\right)^{\frac{3}{2}}=\frac{\left\langle Q_{3}\right\rangle-3\left\langle Q_{2}\right\rangle\left\langle Q_{1}\right\rangle+2\left\langle Q_{1}\right\rangle^{3}}{\left(\left\langle Q_{2}\right\rangle-\left\langle Q_{1}\right\rangle^{2}\right)^{3 / 2}}, \\
K_{\Sigma} & =\frac{\partial^{4} \ln Z}{\partial(a m)^{4}} /\left(\frac{\partial^{2} \ln Z}{\partial(a m)^{2}}\right)^{2} \\
& =\frac{\left\langle Q_{4}\right\rangle-4\left\langle Q_{3}\right\rangle\left\langle Q_{1}\right\rangle-3\left\langle Q_{2}\right\rangle^{2}+12\left\langle Q_{2}\right\rangle\left\langle Q_{1}\right\rangle^{2}-6\left\langle Q_{1}\right\rangle^{4}}{\left(\left\langle Q_{2}\right\rangle-\left\langle Q_{1}\right\rangle^{2}\right)^{2}} .
\end{aligned}
$$

For gluonic observables, gluon action density $G$, plaquette $P$, and Polyakov loop $L, Q_{i}$ is just the $i$ th power of the operator.

\section{SIMULATION DETAILS}

We choose three temporal lattice sizes $N_{t}=4,6,8$ to examine the continuum limit and run simulations for a set of spatial sizes $N_{l}=6-16\left(N_{t}=4\right), 10-24\left(N_{t}=6\right)$, and 12-24 $\left(N_{t}=8\right)$ for finite size scaling studies. Calculations are made with $N_{f}=3$ degenerate flavors of dynamical quarks using the Iwasaki glue [13] and the nonperturbatively $O(a)$-improved Wilson fermion action [14]; i.e., we determine the critical endpoint on the line of $m_{s}=m_{u d}$ $\left(=m_{l}\right)$ on the Columbia phase diagram plot.

We use a highly optimized hybrid Monte Carlo code [15], applying mass preconditioning [16] and rational hybrid Monte Carlo [17], using a second order minimum norm integration scheme [18], putting the pseudofermion action on multiple time scales [19], and employing a minimum residual chronological method [20] to choose the starting guess for the solver. 

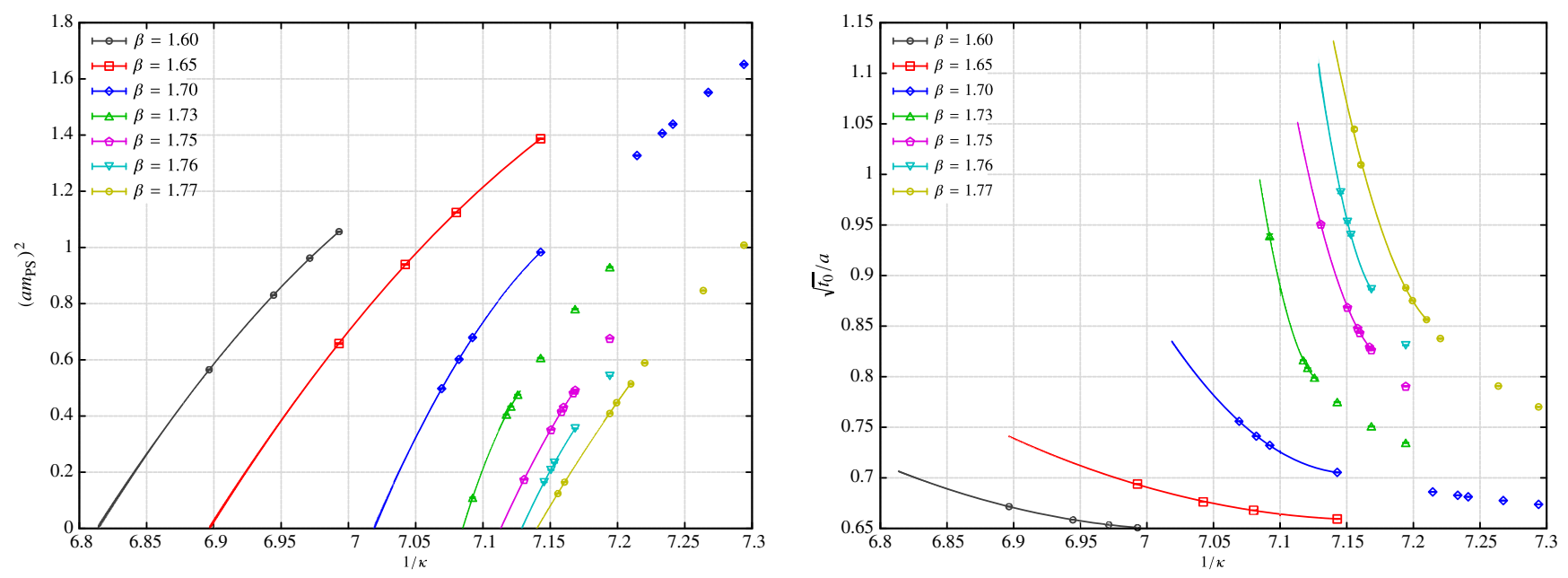

FIG. 2 (color online). (Left panel) $\left(a m_{\mathrm{PS}}\right)^{2}$ vs $1 / \kappa$. (Right panel) $\sqrt{t_{0}} / a$ vs $1 / \kappa$.

We generate $O(100,000)$ trajectories for each lattice parameter set $\left(\beta, \kappa, N_{t}, N_{l}\right)$. We measure the gluon observables $G, P, L$ and cumulants at every trajectory, and the quark observable $\Sigma$ and cumulants at every ten trajectories.
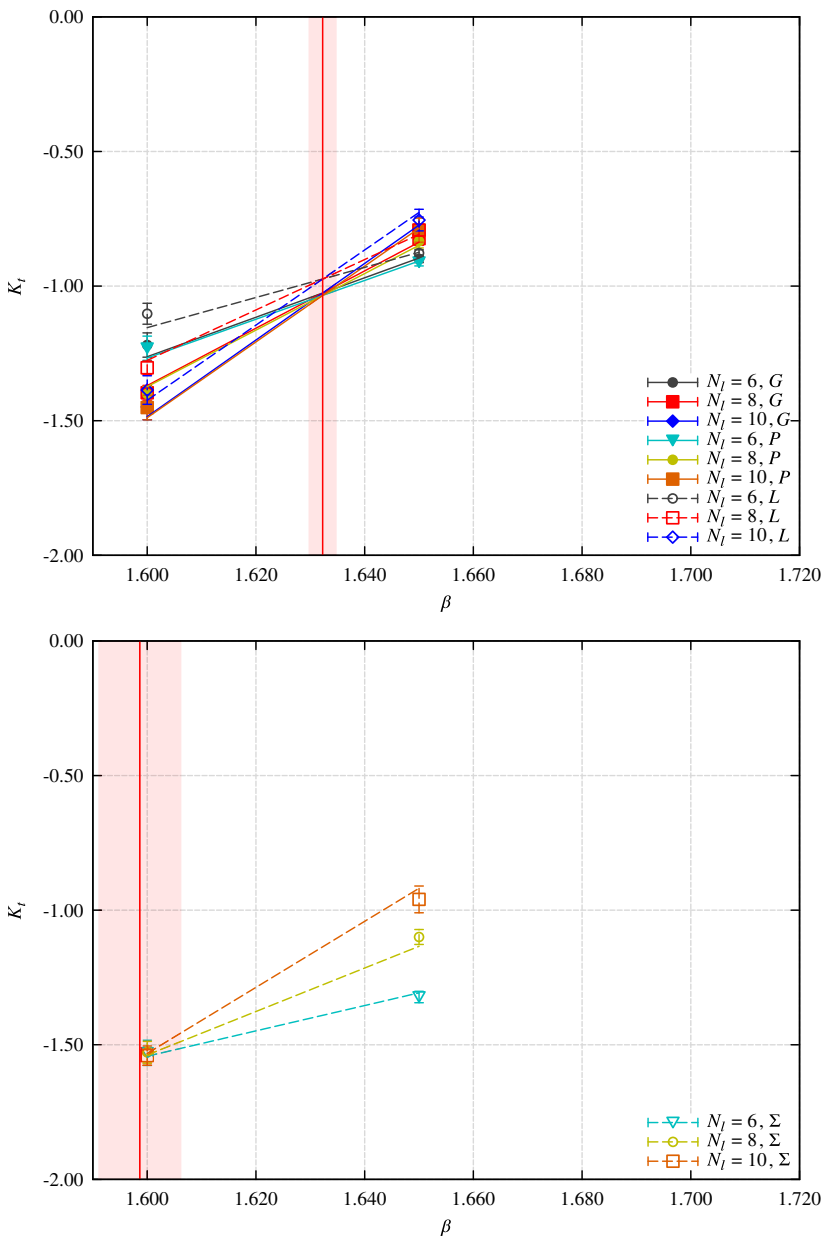

Errors of the observables are estimated by the jackknife method with the bin size of $O(1000)$ trajectories.

To set the physical scale, we perform a set of zero temperature simulations. The simulation parameters,
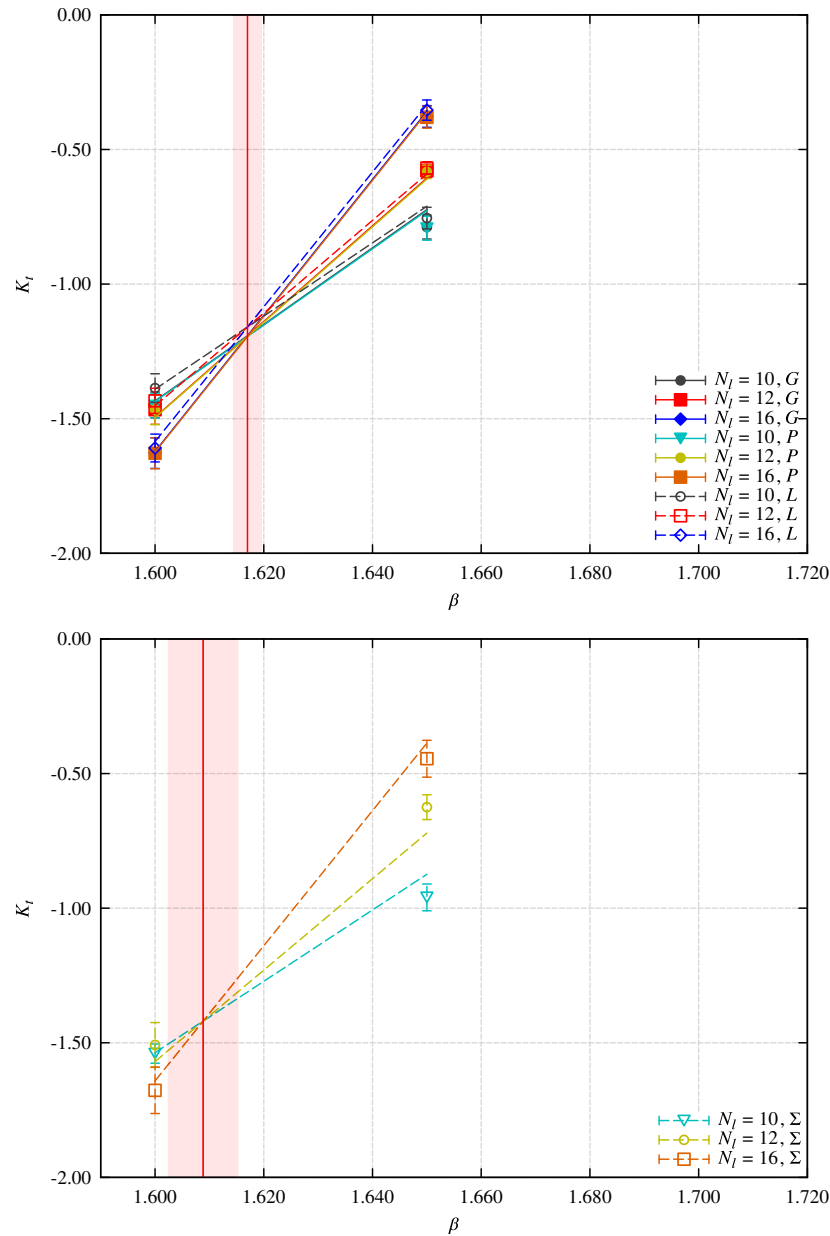

FIG. 3 (color online). Kurtosis intersection for $G, P, L$ on smaller lattices (top-left panel), larger lattices (top-right panel), $\Sigma$ on smaller lattices (bottom-left panel), and larger lattices (bottom-right panel) at $N_{t}=4$. 
results for the mass of the pseudoscalar meson, and the Wilson flow scale parameter $\sqrt{t_{0}} / a$ [21] are summarized in Tables II-VIII in Appendix A. Data are based on $O(500)$ configurations separated by ten trajectories. Errors are estimated by the jackknife method with a bin size of 10 to 100 configurations.

Using a combined fit of form,

$$
\begin{gathered}
\left(a m_{\mathrm{PS}}\right)^{2}=a_{1}\left(\frac{1}{\kappa}-\frac{1}{\kappa_{c}}\right)+a_{2}\left(\frac{1}{\kappa}-\frac{1}{\kappa_{c}}\right)^{2}, \\
\frac{\sqrt{t_{0}}}{a}=b_{0}+b_{1}\left(\frac{1}{\kappa}-\frac{1}{\kappa_{c}}\right)+b_{2}\left(\frac{1}{\kappa}-\frac{1}{\kappa_{c}}\right)^{2},
\end{gathered}
$$

we obtain the critical hopping parameter $\kappa_{c}$ and the expansion coefficients. The fit results are listed in Table IX in Appendix A. We plot data and fits for the pseudoscalar meson mass and the Wilson flow scale in Fig. 2. We tried different fitting forms and ranges, and our final results barely changed within the range of error. The fits presented here give us large $\chi^{2} /$ d.o.f. at $\beta=1.65,1.77$,
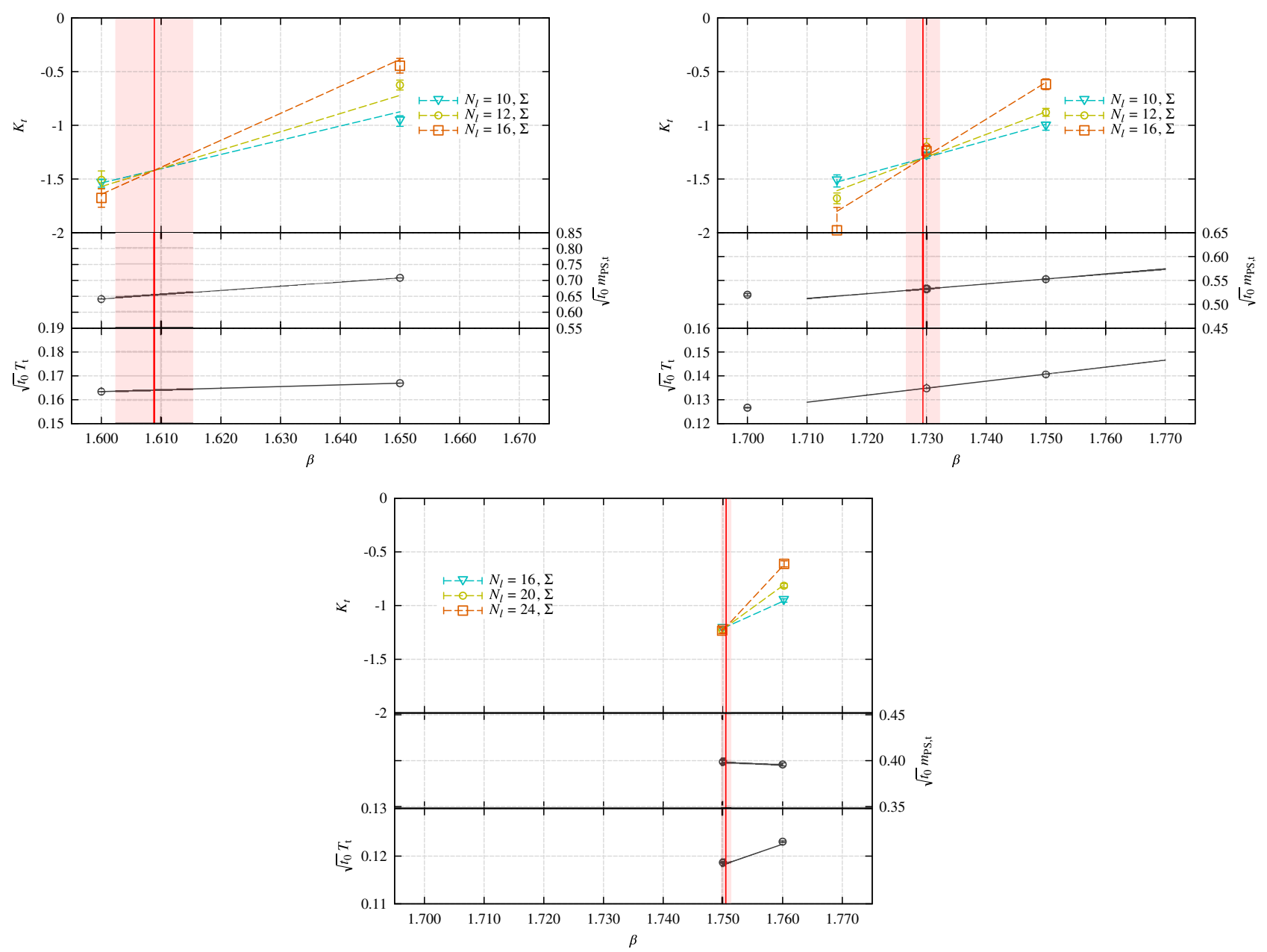

FIG. 4 (color online). $\quad K_{\mathrm{t}}, \sqrt{t_{0}} m_{\mathrm{PS}, \mathrm{t}}, \sqrt{t_{0}} T_{\mathrm{t}}$ vs $\beta$ at $N_{t}=4$ (top-left panel), $N_{t}=6$ (top-right panel), $N_{t}=8$ (bottom panel) determined by the quark observable $\Sigma$.

The first step of our analysis is to locate the position of thermal transition on the $(\beta, \kappa)$ plane for each $N_{t}$ and $N_{l}$. In Appendix $\mathrm{C}$ we show representative results of susceptibility and kurtosis for the four observables at $N_{t}=4$ (Figs. 9 and 10), $N_{t}=6$ (Figs. 11 and 12), and $N_{t}=8$ (Figs. 13 and 14). The pairs of figures are chosen so that the pair sandwiches the critical endpoint.

For $N_{t}=4$ and 6 , we fix $\beta$ and make measurements at several values of $\kappa$. In order to locate the transition point,

although data points appear to lie on the fitting curves. This
is because we are trying to fit a wide range with too few data points of small statistical errors. Our conclusion, however, is unaffected since our final analysis excludes mass and the temperature at $\beta=1.77$ are not used in our interpolation, and those at $\beta=1.65$ are only used for $N_{t}=4$, which does not enter into our continuum extrapolation, as we will see in Sec. V.

\section{RESULTS}

\section{A. Location of finite temperature transition}



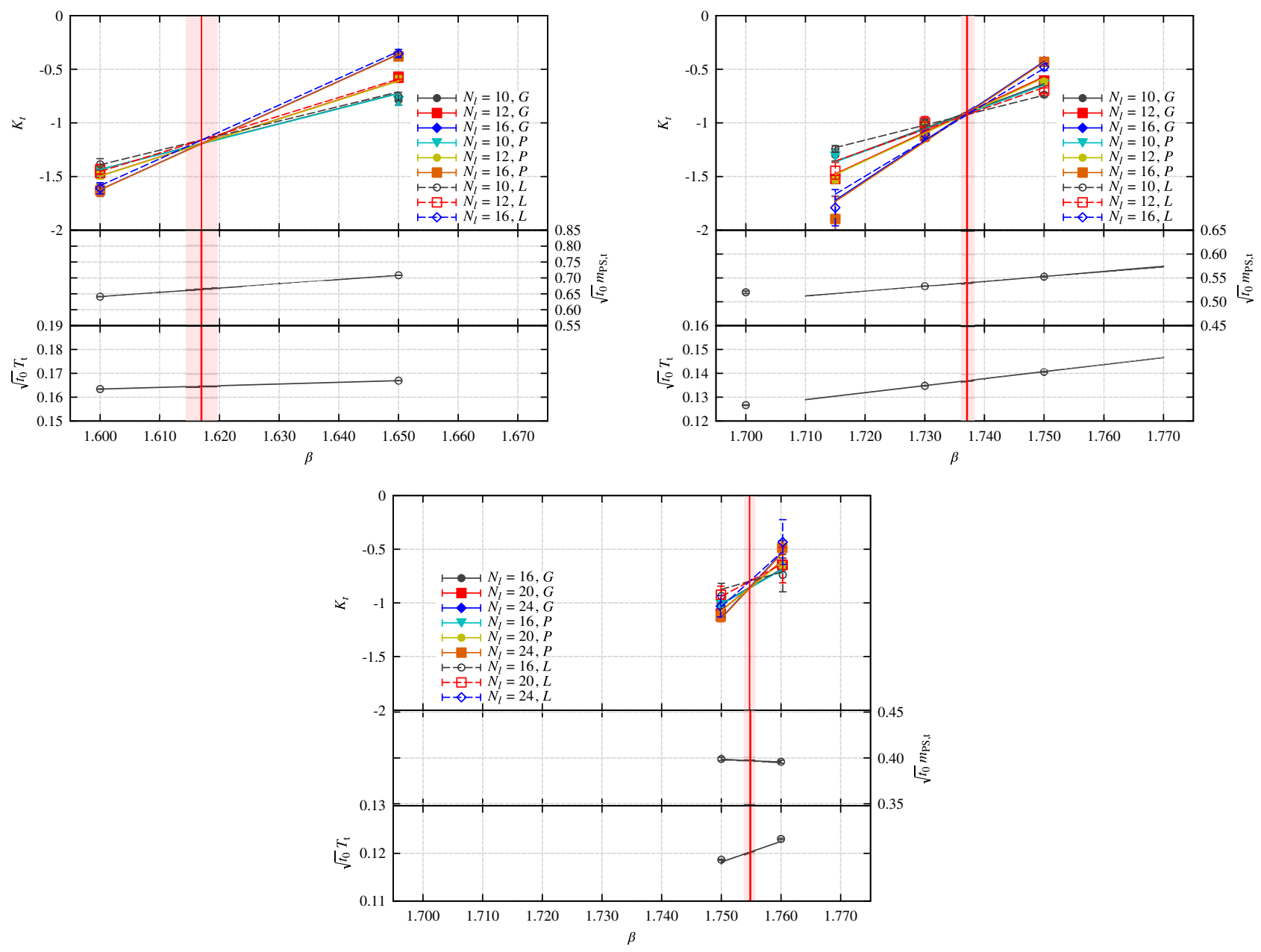

FIG. 5 (color online). $\quad K_{\mathrm{t}}, \sqrt{t_{0}} m_{\mathrm{PS}, \mathrm{t}}, \sqrt{t_{0}} T_{\mathrm{t}}$ vs $\beta$ at $N_{t}=4$ (top-left panel), $N_{t}=6$ (top-right panel), and $N_{t}=8$ (bottom panel) determined by the gluon observables $G, P, L$.

susceptibility and kurtosis are fitted with a quadratic ansatz in $\kappa$. As one observes from the fit curves, the maximum of susceptibility and the minimum of kurtosis are mutually consistent. Though not shown, we find that the zero of skewness obtained by a linear fit is consistent as well. We choose the maximum of susceptibility to be the point of transition. The results for the value of $\kappa$ thus defined are listed in Table X in Appendix B. The errors are calculated from the fit.

For $N_{t}=8$, we fix $\kappa$ and make runs at several values of $\beta$. Since these runs are computationally expensive, we carry out long runs at one or two judiciously chosen values of $\beta$ close to the would-be transition point and make single- or multiensemble reweighting to search for the maximum susceptibility, as shown in Figs. 13 and 14 in Appendix C. The values of $\beta$ corresponding to the susceptibility peak are listed in Table XI. The central values and errors are calculated by estimating the errors of reweighted values by the jackknife method with bin size of 100 trajectories.

Having determined the location of thermal transition from the peak of susceptibility, we calculate the value of kurtosis at the transition point with the help of the fit or the reweighting of kurtosis. The results are listed in Tables XII and XIII in Appendix B.

\section{B. Kurtosis intersection analysis}

Ideally, if one plots the value of kurtosis along the transition line with a given temporal size $N_{t}$, the curves for various spatial sizes $N_{l}$ intersect at a single point corresponding to the critical endpoint. In practice, the intersection point varies for various combinations of $N_{l}$ 's and also depends on the observables. This is illustrated in Fig. 3, where we separately plot $N_{t}=4$ results for the gluon observables $G, P, L$ for smaller spatial sizes $N_{l}=6,8,10$ and for larger sizes $N_{l}=10,12,16$, and similarly for the quark observable $\Sigma$. We find that the gluon observable and the quark observable give rather different estimates for the intersection point $\beta_{\mathrm{E}}$ at smaller values of $N_{l}$ which, however, become consistent for larger values of $N_{l}$.

We suspect that the situation above is due to the fact that our observables are linear combinations of energy and magnetization operators with different mixing coefficients, so that the subleading corrections of $O\left(L^{y_{t}-y_{h}}\right)$ differ in 
TABLE I. Fit results for the kurtosis intersection point and interpolated values of $\sqrt{t_{0}} m_{\mathrm{PS}}$ and $\sqrt{t_{0}} T$. The top table shows values obtained with quark observable $\Sigma$. The bottom table is obtained from the gluon observables $G, P, L$. We also list the value of $\kappa_{E}$ for completeness.

\begin{tabular}{|c|c|c|c|c|c|c|c|c|}
\hline \multicolumn{9}{|c|}{ Analysis with quark observable $\Sigma$} \\
\hline$\overline{N_{t}}$ & $\beta_{\mathrm{E}}$ & $\kappa_{E}$ & $K_{\mathrm{E}}$ & $\nu$ & $a$ & $\chi^{2} /$ d.o.f. & $\sqrt{t_{0}} m_{\mathrm{PS}, \mathrm{E}}$ & $\sqrt{t_{0}} T_{\mathrm{E}}$ \\
\hline$\overline{4}$ & $1.6089(66)$ & $0.14305(30)$ & $-1.42(11)$ & $0.74(15)$ & $0.58(39)$ & 4.401 & $0.6530(88)$ & $0.16402(47)$ \\
\hline 6 & $1.7294(29)$ & $0.14045(11)$ & $-1.307(57)$ & $0.58(11)$ & $0.30(24)$ & 1.145 & $0.5318(30)$ & $0.13457(84)$ \\
\hline 8 & $1.75054(87)$ & $0.140226(25)$ & $-1.198(33)$ & $0.465(58)$ & $0.065(53)$ & 0.148 & $0.3986(16)$ & $0.11890(40)$ \\
\hline \multicolumn{9}{|c|}{ Analysis with gluon observables $G, P, L$} \\
\hline$\overline{N_{t}}$ & $\beta_{\mathrm{E}}$ & $\kappa_{E}$ & $K_{\mathrm{E}}$ & $\nu$ & $a$ & $\chi^{2} /$ d.o.f. & $\sqrt{t_{0}} m_{\mathrm{PS}, \mathrm{E}}$ & $\sqrt{t_{0}} T_{\mathrm{E}}$ \\
\hline 4 & $1.6170(27)$ & $0.14274(44)$ & $\begin{array}{l}-1.192(54) \\
-1.195(54) \\
-1.160(54)\end{array}$ & $\begin{array}{l}0.82(13) \\
0.81(13) \\
0.77(11)\end{array}$ & $\begin{array}{l}0.85(41) \\
0.84(40) \\
0.68(33)\end{array}$ & 1.313 & $0.6639(37)$ & $0.16460(19)$ \\
\hline 6 & $1.7372(12)$ & $0.140163(44)$ & $\begin{array}{l}-0.900(31) \\
-0.910(31) \\
-0.920(25)\end{array}$ & $\begin{array}{c}0.81(13) \\
0.79(12) \\
0.536(63)\end{array}$ & $\begin{array}{l}1.20(56) \\
1.11(52) \\
0.19(11)\end{array}$ & 1.469 & $0.5396(14)$ & $0.13685(35)$ \\
\hline 8 & $1.75474(96)$ & $0.140106(28)$ & $\begin{array}{l}-0.856(40) \\
-0.863(40) \\
-0.799(63)\end{array}$ & $\begin{array}{l}0.65(17) \\
0.64(17) \\
0.35(30)\end{array}$ & $\begin{array}{c}0.43(52) \\
0.43(51) \\
0.006(43)\end{array}$ & 0.684 & $0.3974(12)$ & $0.12073(43)$ \\
\hline
\end{tabular}

magnitude from observable to observable, especially between gluon and quark observables. Since we do not have enough data sets to resolve the subleading contributions, we use the quark observable $\Sigma$ as our primary operator and employ gluon observables to estimate the systematic errors in the results.

In Fig. 4 we show the intersection analysis using $\Sigma$. Also plotted is the determination of the pseudoscalar meson mass $m_{\mathrm{PS}, \mathrm{E}}$ and the transition temperature $T_{\mathrm{E}}$ at the critical endpoint in units of the Wilson flow scale $\sqrt{t_{0}}$. Figure 5

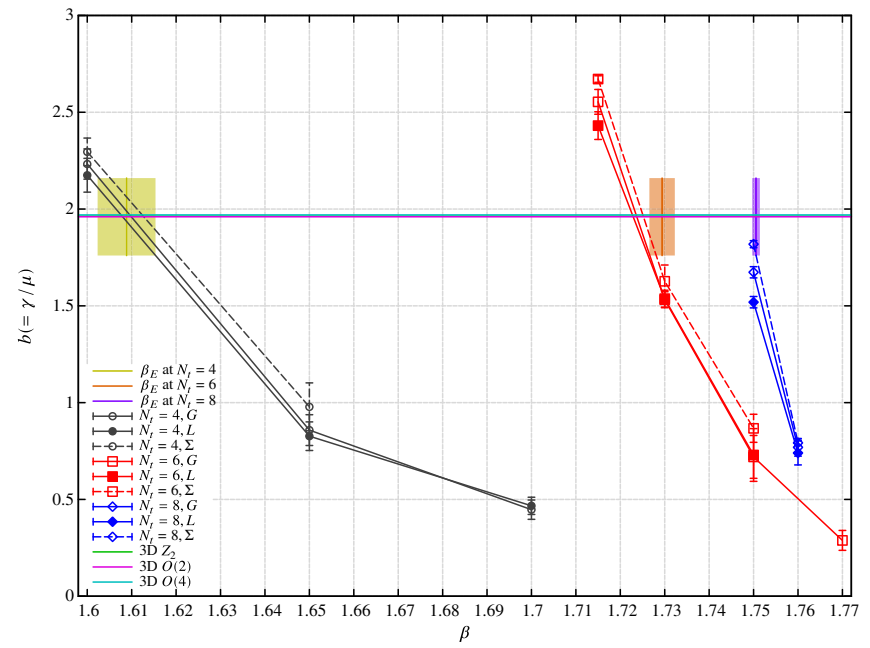

FIG. 6 (color online). Susceptibility exponent $b$ as a function of $\beta$. The horizontal line corresponds to the value $\gamma / \nu=1.964$ for $\mathrm{Z}(2)$ universality in three dimensions. Colored blocks superimposed are estimates of the critical endpoint $\beta_{E}$ obtained in the kurtosis intersection analysis. shows similar plots for gluon observables. The numerical values of the estimated critical endpoint $\left(\beta_{E}, \kappa_{E}\right)$, kurtosis at that point $K_{\mathrm{E}}$, the exponent $\nu$, the pseudoscalar meson mass $\sqrt{t_{0}} m_{\mathrm{PS}, \mathrm{E}}$, and the critical temperature $\sqrt{t_{0}} T_{\mathrm{E}}$ are listed in Table I.

We observe in this table that the kurtosis $K_{\mathrm{E}}$ and the exponent $\nu$ show significant variation, depending on the choice of observables, particularly with $K_{\mathrm{E}}$. This, of course, should not be so, and we likely need larger spatial sizes to make certain that infinite volume values are attained.

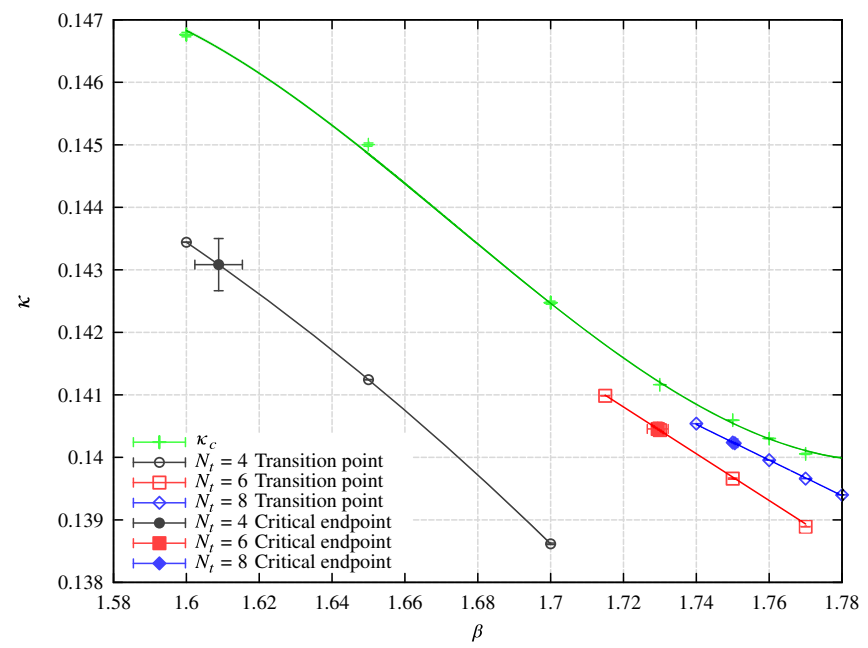

FIG. 7 (color online). Phase diagram of $N_{f}=3$ lattice QCD on the $(\beta, \kappa)$ plane. An estimation of the line of first order phase transition ending at a critical point marked by full circles is shown for $N_{t}=4,6,8$, together with the line of $\kappa_{c}$ where pion mass vanishes. 

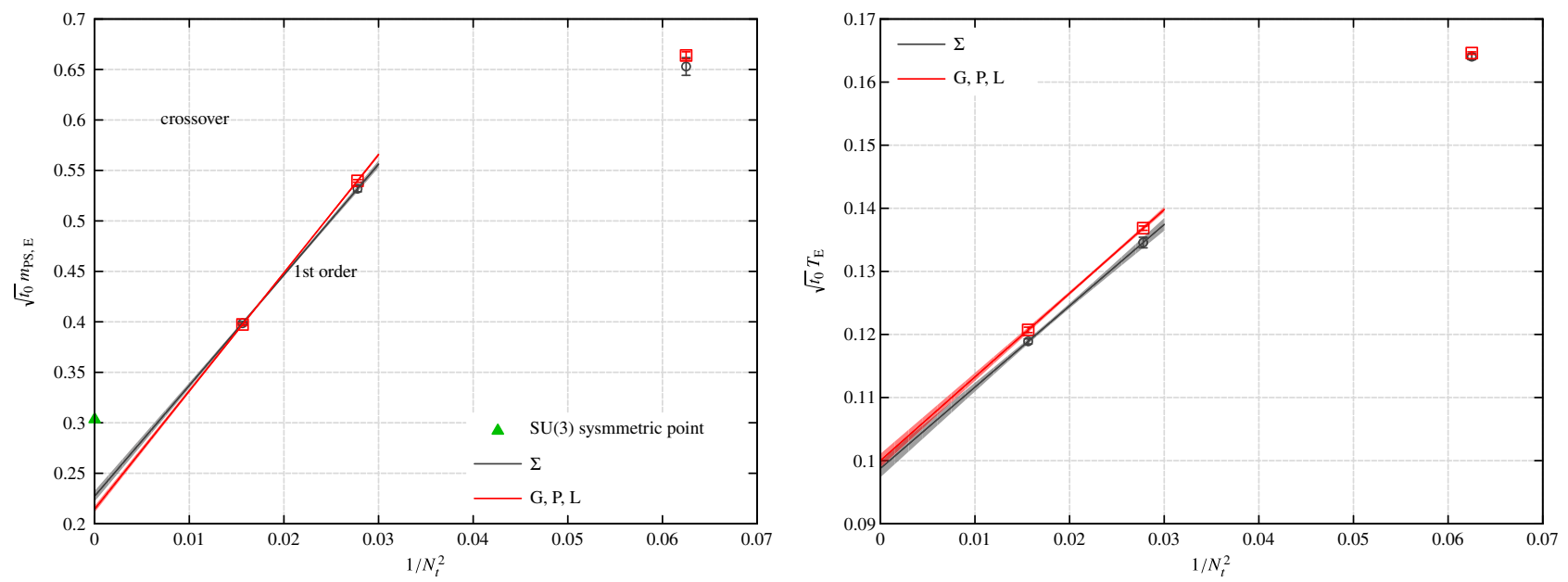

FIG. 8 (color online). Continuum extrapolation of $\sqrt{t_{0}} m_{\mathrm{PS}, \mathrm{E}}$ and $\sqrt{t_{0}} T_{\mathrm{E}}$ as a function of $1 / N_{t}^{2}$.

Comparing the results with the values of possible universality classes, i.e., $K_{\mathrm{E}}=-1.396$ and $\nu=0.630$ for $\mathrm{Z}(2)$, $K_{\mathrm{E}}=-1.758$ and $\nu=0.672$ for $\mathrm{O}(2)$, and $K_{\mathrm{E}}=-1.908$ and $\nu=0.748$ for $\mathrm{O}(4)$, our numbers favor $\mathrm{Z}(2)$.

We may attempt to check the location of the critical endpoint and the universality class by looking at the spatial size variation of the peak height of susceptibility:

$$
\chi^{\max }=a N_{l}^{b} .
$$

The exponent $b$ should equal $\gamma / \nu$ at the critical endpoint; $b=d$, the space dimension, at the first order side at lower $\beta$; and $b=0$ at larger $\beta$ after the phase transition terminates. We plot the exponent $b$ as a function of $\beta$ for each $N_{t}$ in Fig. 6. The horizontal line corresponds to the value $\gamma / \nu=1.964$ for $\mathrm{Z}(2)$. Superimposed blocks on the line are the estimate of $\beta_{E}$ from the kurtosis intersection analysis in Table I. We observe consistency with the crossing point of the exponent $b$ with the horizontal line. Unfortunately, $\gamma / \nu$ has a similar value $\gamma / \nu=1.964,1.962,1.975$ for $Z(2), O$ (2), and $\mathrm{O}(4)$. Distinguishing $\mathrm{Z}(2)$ from the other universality classes would be difficult.

In Fig. 7 , we show the phase diagram in the $(\beta, \kappa)$ plane. Open symbols are the location of thermal transition as determined by the susceptibility peak analysis, and filled symbols are the location of the critical endpoint estimated by the kurtosis intersection analysis for $N_{t}=4,6,8$. Lines are a smooth interpolation of the thermal phase transition line; the segment to the left of the filled symbols corresponds to the first order transition and the segment to the right corresponds to crossover. The location of the critical hopping parameter $\kappa_{c}$ where pion mass vanishes is also shown.

\section{Continuum estimation of $m_{\mathrm{PS}}$ and $T$ at the critical endpoint}

Finally, we extrapolate $\sqrt{t_{0}} m_{\mathrm{PS}, \mathrm{E}}$ and $\sqrt{t_{0}} T_{\mathrm{E}}$ to the continuum limit. In Fig. 8 we plot $\sqrt{t_{0}} m_{\mathrm{PS}, \mathrm{E}}$ and $\sqrt{t_{0}} T_{\mathrm{E}}$ against $1 / N_{t}^{2}$. Results based on the quark and gluon estimations of $\beta_{E}$ are both shown. The green triangle on the $y$ axis denotes the $\mathrm{SU}(3)$ symmetric point estimated by $\sqrt{t_{0}} \sqrt{\left(m_{\pi}^{2}+2 m_{K}^{2}\right) / 3} \sim 0.305$. We have used the value $1 / \sqrt{t_{0}}=1.347(30) \mathrm{GeV}$ [22] (see also [23]).

It is clear that $N_{t}=4$ is outside the scaling region. We therefore make a linear extrapolation with the points for $N_{t}=6$ and 8 to obtain

$$
\begin{aligned}
\sqrt{t_{0}} m_{\mathrm{PS}, \mathrm{E}} & =0.2274(52)(108), \\
\sqrt{t_{0}} T_{\mathrm{E}} & =0.0988(14)(9),
\end{aligned}
$$

where the first error is statistical error and the second error is systematic error estimated from the spread between the determinations with quark and gluon observables. Normalizing by $m_{\mathrm{PS}}^{\text {phys,sym }} \equiv \sqrt{\left(m_{\pi}^{2}+2 m_{K}^{2}\right) / 3}$, we obtain

$$
\frac{m_{\mathrm{PS}, \mathrm{E}}}{m_{\mathrm{PS}}^{\text {physym }}}=0.745(17)(35)(17),
$$

where the third error comes from error of $\sqrt{t_{0}}$. Converting to physical units, we estimate $T_{\mathrm{E}}=133(2)(1)(3) \mathrm{MeV}$ and $m_{\mathrm{PS}, \mathrm{E}}=306(7)(14)(7) \mathrm{MeV}$.

\section{SUMMARY}

We have investigated the critical endpoint of QCD with $N_{f}=3$ dynamical flavors of nonperturbatively $O(a)$ improved Wilson fermions. We have determined the critical endpoint by using the intersection points of kurtosis at the temporal sizes $N_{t}=4,6,8$.

The values of kurtosis $K_{E}$ at the critical endpoint and the exponent $\nu$ obtained from the intersection analysis favor the $\mathrm{Z}(2)$ universality class, and the location of the critical endpoint is consistent with those estimated from the susceptibility analysis. Further work with larger spatial 
lattice sizes would be needed, however, to establish the universality class.

Our current estimate of the pseudoscalar meson mass at the critical endpoint in the continuum limit is about $25 \%$ lighter than the flavor $\mathrm{SU}(3)$ symmetric point at the quark mass $m_{q}=\left(m_{u}^{\text {phy }}+m_{d}^{\text {phy }}+m_{s}^{\text {phy }}\right) / 3$. This suggests the possibility that the quark mass at the critical endpoint is not as small as suggested by recent studies with improved staggered quark action.

Our continuum extrapolation has been made, however, only with two temporal lattice sizes, $N_{t}=6$ and 8 , as we have found that $N_{t}=4$ is out of the region of $1 / N_{t}^{2}$ linear scaling. We are planning further studies at larger temporal sizes to obtain conclusive results.

\section{ACKNOWLEDGMENTS}

The Berlin QCD code [15] was used in this work. Our calculations were carried out on the $\mathrm{K}$ computer provided by the RIKEN Advanced Institute for Computational Science, HA8000 and FX10 at the University of Tokyo, HA8000-tc/HT210 and FX10 at Kyushu University, and Cray XC30 with Xeon Phi at Kyoto University. This research came about through the HPCI System Research Project (Project No. hp120115), FX10 at the University of Tokyo and Kyushu University, HA8000 at the University of Tokyo through the HPCI System Research Project (Project No. hp130092), and System E at Kyoto University through the HPCI System Research Project (Project No. hp140180). This work was supported by Large Scale Simulation Program Grants No. 12/13-13 (FY2012-2013) and No. 13/14-12 (FY2013-2014) of the High Energy Accelerator Research Organization (KEK). Part of this work was supported by the Grants-in-Aid for Scientific Research from the Ministry of Education, Culture, Sports, Science and Technology (Grant No. 26800130).

\section{APPENDIX A: WILSON FLOW SCALE AND PSEUDOSCALAR MESON MASS AT ZERO TEMPERATURE}

Simulation parameters, results for the mass of the pseudoscalar meson $a m_{\mathrm{PS}}$, and the Wilson flow scale parameter $\sqrt{t_{0}} / a$ [21] are summarized in Tables II-VIII. The results of fitting data to (18) and (19) as a function of $\kappa$ for each $\beta$ are given in Table IX.

TABLE II. Simulation parameters $\kappa, N_{l}, N_{t}$, and $\sqrt{t_{0}} / a$ and $a m_{\mathrm{PS}}$ at $\beta=1.60$.

\begin{tabular}{lllll}
\hline \hline$\kappa$ & $N_{l}$ & $N_{t}$ & \multicolumn{1}{c}{$\sqrt{t_{0}} / a$} & \multicolumn{1}{c}{$a m_{\mathrm{PS}}$} \\
\hline 0.143000 & 12 & 24 & $0.650783(71)$ & $1.02752(71)$ \\
0.143446 & 12 & 24 & $0.653722(72)$ & $0.98078(68)$ \\
0.144000 & 12 & 24 & $0.658485(90)$ & $0.91122(74)$ \\
0.145000 & 12 & 24 & $0.67160(15)$ & $0.7516(13)$ \\
\hline \hline
\end{tabular}

TABLE III. Simulation parameters $\kappa, N_{l}, N_{t}$, and $\sqrt{t_{0}} / a$ and $a m_{\mathrm{PS}}$ at $\beta=1.65$.

\begin{tabular}{lllll}
\hline \hline$\kappa$ & $N_{l}$ & $N_{t}$ & \multicolumn{1}{c}{$\sqrt{t_{0}} / a$} & \multicolumn{1}{c}{$a m_{\mathrm{PS}}$} \\
\hline 0.140000 & 12 & 24 & $0.659353(65)$ & $1.17770(72)$ \\
0.141240 & 12 & 24 & $0.66818(11)$ & $1.06023(80)$ \\
0.142000 & 12 & 24 & $0.67631(11)$ & $0.96934(88)$ \\
0.143000 & 12 & 24 & $0.69391(21)$ & $0.8111(14)$ \\
\hline \hline
\end{tabular}

TABLE IV. Simulation parameters $\kappa, N_{l}, N_{t}$, and $\sqrt{t_{0}} / a$ and $a m_{\mathrm{PS}}$ at $\beta=1.70$.

\begin{tabular}{lllll}
\hline \hline$\kappa$ & $N_{l}$ & $N_{t}$ & \multicolumn{1}{c}{$\sqrt{t_{0}} / a$} & \multicolumn{1}{c}{$a m_{\mathrm{PS}}$} \\
\hline 0.137100 & 12 & 24 & $0.673734(84)$ & $1.28517(96)$ \\
0.137600 & 12 & 24 & $0.67752(11)$ & $1.2457(10)$ \\
0.138100 & 12 & 24 & $0.68124(11)$ & $1.1995(11)$ \\
0.138250 & 12 & 24 & $0.68277(12)$ & $1.18585(85)$ \\
0.138610 & 12 & 24 & $0.68608(12)$ & $1.15202(69)$ \\
0.140000 & 16 & 32 & $0.705367(99)$ & $0.99132(64)$ \\
0.141000 & 16 & 32 & $0.73207(14)$ & $0.8243(15)$ \\
0.141200 & 16 & 32 & $0.74115(22)$ & $0.7758(11)$ \\
0.141456 & 16 & 32 & $0.75598(23)$ & $0.7055(12)$ \\
\hline \hline
\end{tabular}

TABLE V. Simulation parameters $\kappa, N_{l}, N_{t}$, and $\sqrt{t_{0}} / a$ and $a m_{\mathrm{PS}}$ at $\beta=1.73$.

\begin{tabular}{lllll}
\hline \hline$\kappa$ & $N_{l}$ & $N_{t}$ & \multicolumn{1}{c}{$\sqrt{t_{0}} / a$} & \multicolumn{1}{c}{$a m_{\mathrm{PS}}$} \\
\hline 0.139000 & 12 & 24 & $0.73453(26)$ & $0.96412(97)$ \\
0.139500 & 12 & 24 & $0.75087(27)$ & $0.8833(11)$ \\
0.140000 & 16 & 32 & $0.77484(31)$ & $0.7787(11)$ \\
0.140334 & 16 & 32 & $0.79915(38)$ & $0.68974(85)$ \\
0.140435 & 16 & 32 & $0.80879(42)$ & $0.65851(99)$ \\
0.140500 & 16 & 32 & $0.81630(36)$ & $0.63650(93)$ \\
0.141000 & 16 & 32 & $0.9391(21)$ & $0.3306(45)$ \\
\hline \hline
\end{tabular}

TABLE VI. Simulation parameters $\kappa, N_{l}, N_{t}$, and $\sqrt{t_{0}} / a$ and $a m_{\mathrm{PS}}$ at $\beta=1.75$.

\begin{tabular}{lllll}
\hline \hline$\kappa$ & $N_{l}$ & $N_{t}$ & \multicolumn{1}{c}{$\sqrt{t_{0}} / a$} & \multicolumn{1}{c}{$a m_{\mathrm{PS}}$} \\
\hline 0.139000 & 12 & 24 & $0.79055(42)$ & $0.82237(93)$ \\
0.139500 & 12 & 24 & $0.82671(76)$ & $0.7017(15)$ \\
0.139529 & 16 & 32 & $0.82959(42)$ & $0.69470(91)$ \\
0.139669 & 16 & 32 & $0.84360(53)$ & $0.6569(11)$ \\
0.139700 & 16 & 32 & $0.84799(45)$ & $0.64517(96)$ \\
0.139850 & 16 & 32 & $0.86861(51)$ & $0.59293(89)$ \\
0.140242 & 16 & 32 & $0.9508(10)$ & $0.4176(18)$ \\
\hline \hline
\end{tabular}

TABLE VII. Simulation parameters $\kappa, N_{l}, N_{t}$, and $\sqrt{t_{0}} / a$ and $a m_{\mathrm{PS}}$ at $\beta=1.76$.

\begin{tabular}{lllll}
\hline \hline$\kappa$ & $N_{l}$ & $N_{t}$ & \multicolumn{1}{c}{$\sqrt{t_{0}} / a$} & \multicolumn{1}{c}{$a m_{\mathrm{PS}}$} \\
\hline 0.139000 & 16 & 32 & $0.83107(33)$ & $0.73691(77)$ \\
0.139500 & 16 & 32 & $0.88650(51)$ & $0.59667(93)$ \\
0.139800 & 16 & 32 & $0.94019(98)$ & $0.4839(14)$ \\
0.139850 & 16 & 32 & $0.9530(12)$ & $0.4567(17)$ \\
0.139950 & 16 & 32 & $0.9823(13)$ & $0.4060(14)$ \\
\hline \hline
\end{tabular}


TABLE VIII. Simulation parameters $\kappa, N_{l}, N_{t}$, and $\sqrt{t_{0}} / a$ and $a m_{\mathrm{PS}}$ at $\beta=1.77$.

\begin{tabular}{lllll}
\hline \hline$\kappa$ & $N_{l}$ & $N_{t}$ & $\sqrt{t_{0}} / a$ & $1.0040(12)$ \\
\hline 0.137100 & 12 & 24 & $0.77014(39)$ & $0.91999(86)$ \\
0.137670 & 12 & 24 & $0.79076(35)$ & $0.7675(12)$ \\
0.138500 & 12 & 24 & $0.83773(53)$ & $0.7172(18)$ \\
0.138700 & 12 & 24 & $0.85652(79)$ & $0.66902(80)$ \\
0.138903 & 16 & 32 & $0.87524(52)$ & $0.63966(81)$ \\
0.139000 & 16 & 32 & $0.88795(57)$ & $0.4063(14)$ \\
0.139653 & 16 & 32 & $1.0096(13)$ & $0.3528(20)$ \\
0.139750 & 16 & 32 & $1.0447(17)$ & \\
\hline \hline
\end{tabular}

TABLE IX. Fit results to (18) and (19) for critical hopping parameter $\kappa_{c}$ and coefficients for pseudoscalar meson mass $a m_{\mathrm{PS}}$ and Wilson flow parameter $\sqrt{t_{0}} / a$ for $\beta=1.60$ to 1.77 .

\begin{tabular}{|c|c|c|c|c|c|c|c|c|}
\hline$\beta$ & $\kappa_{c}$ & $a_{1}$ & $a_{2}$ & $b_{0}$ & $b_{1}$ & $b_{2}$ & $\chi^{2} /$ d.o.f. & fit range $\kappa>$ \\
\hline 1.60 & $0.146763(36)$ & $7.61(17)$ & $-9.57(74)$ & $0.7064(13)$ & $-0.516(14)$ & $1.149(46)$ & 2.18 & 0.1430 \\
\hline 1.65 & $0.145000(29)$ & $7.60(11)$ & $-8.01(35)$ & $0.7409(12)$ & $-0.5967(99)$ & $1.079(25)$ & 27.55 & 0.1400 \\
\hline 1.70 & $0.142475(26)$ & $11.16(29)$ & $-26.1(2.0)$ & $0.8347(28)$ & $-1.917(38)$ & $7.05(18)$ & 2.32 & 0.1400 \\
\hline 1.73 & $0.1411461(64)$ & $15.62(34)$ & $-98.1(7.5)$ & $0.9944(42)$ & $-8.17(21)$ & $83.2(3.2)$ & 0.90 & 0.1403 \\
\hline 1.75 & $0.1405834(88)$ & $10.55(25)$ & $-29.0(3.8)$ & $1.0511(41)$ & $-6.60(15)$ & 46.1(1.8) & 1.97 & 0.1395 \\
\hline 1.76 & $0.140276(17)$ & $10.56(62)$ & $-40(12)$ & $1.109(12)$ & $-9.06(65)$ & $87(11)$ & 1.38 & 0.1395 \\
\hline 1.77 & $0.1400560(95)$ & $8.21(25)$ & $-11.7(3.4)$ & $1.1315(41)$ & $-6.47(14)$ & $36.2(1.5)$ & 7.74 & 0.1387 \\
\hline
\end{tabular}

\section{APPENDIX B: TRANSITION POINT AND KURTOSIS}

The estimated transition point and the value of kurtosis at transition point $K_{\mathrm{t}}$ are summarized in Tables X-XIII.

TABLE X. Transition point for each observable at $N_{t}=4,6$.

\begin{tabular}{|c|c|c|c|c|c|c|}
\hline$N_{t}$ & $N_{l}$ & $\beta$ & $\kappa_{\mathrm{t}, P}$ & $\kappa_{\mathrm{t}, G}$ & $\kappa_{\mathrm{t}, L}$ & $\kappa_{\mathrm{t}, \Sigma}$ \\
\hline 4 & 6 & 1.60 & $0.1434492(75)$ & $0.1434487(75)$ & $0.1434445(86)$ & $0.1434543(77)$ \\
\hline 4 & 8 & 1.60 & $0.1434481(48)$ & $0.1434478(48)$ & $0.1434466(48)$ & $0.1434512(48)$ \\
\hline 4 & 10 & 1.60 & $0.1434485(36)$ & $0.1434484(36)$ & $0.1434473(36)$ & $0.1434499(37)$ \\
\hline 4 & 12 & 1.60 & $0.1434386(36)$ & $0.1434385(36)$ & $0.1434379(36)$ & $0.1434404(30)$ \\
\hline 4 & 16 & 1.60 & $0.1434392(41)$ & $0.1434392(41)$ & $0.1434387(42)$ & $0.1434412(40)$ \\
\hline 4 & 6 & 1.65 & $0.1412184(94)$ & $0.1412158(96)$ & $0.1412102(96)$ & $0.141260(16)$ \\
\hline 4 & 8 & 1.65 & $0.1412541(56)$ & $0.1412529(56)$ & $0.1412450(53)$ & $0.1412695(58)$ \\
\hline 4 & 10 & 1.65 & $0.1412430(45)$ & $0.1412426(46)$ & $0.1412381(54)$ & $0.1412479(43)$ \\
\hline 4 & 12 & 1.65 & $0.1412321(67)$ & $0.1412314(69)$ & $0.1412266(76)$ & $0.1412394(80)$ \\
\hline 4 & 16 & 1.65 & $0.1412416(25)$ & $0.1412413(26)$ & $0.1412379(30)$ & $0.1412433(36)$ \\
\hline 4 & 6 & 1.70 & $0.138633(11)$ & $0.138627(11)$ & $0.1386224(94)$ & \\
\hline 4 & 8 & 1.70 & $0.138636(21)$ & $0.138631(20)$ & $0.138615(18)$ & \\
\hline 4 & 10 & 1.70 & $0.1386188(99)$ & $0.1386157(98)$ & $0.1386081(84)$ & \\
\hline 6 & 10 & 1.715 & $0.1409862(28)$ & $0.1409861(28)$ & $0.1409945(26)$ & $0.1409900(26)$ \\
\hline 6 & 12 & 1.715 & $0.1409867(16)$ & $0.1409867(16)$ & $0.1409893(15)$ & $0.1409881(17)$ \\
\hline 6 & 16 & 1.715 & $0.1409853(12)$ & $0.1409853(12)$ & $0.1409860(12)$ & $0.1409856(12)$ \\
\hline 6 & 10 & 1.73 & $0.1404250(90)$ & $0.1404248(93)$ & $0.1404360(38)$ & $0.1404295(55)$ \\
\hline 6 & 12 & 1.73 & $0.1404333(46)$ & $0.1404333(45)$ & $0.1404389(64)$ & $0.1404367(69)$ \\
\hline 6 & 16 & 1.73 & $0.1404347(17)$ & $0.1404346(17)$ & $0.1404365(17)$ & $0.1404361(17)$ \\
\hline 6 & 24 & 1.73 & $0.14043436(53)$ & $0.14043435(53)$ & $0.14043496(52)$ & $0.14043387(80)$ \\
\hline 6 & 10 & 1.75 & $0.1396579(92)$ & $0.1396575(94)$ & $0.1396804(44)$ & $0.1396564(85)$ \\
\hline 6 & 12 & 1.75 & $0.1396607(72)$ & $0.1396605(74)$ & $0.1396777(68)$ & $0.1396673(69)$ \\
\hline 6 & 16 & 1.75 & $0.1396588(81)$ & $0.1396584(82)$ & $0.1396716(42)$ & $0.1396676(41)$ \\
\hline 6 & 10 & 1.77 & $0.1388967(85)$ & $0.1388958(87)$ & $0.1389364(98)$ & \\
\hline 6 & 12 & 1.77 & $0.1388934(49)$ & $0.1388928(49)$ & $0.1389263(61)$ & \\
\hline 6 & 16 & 1.77 & $0.1388942(46)$ & $0.1388937(47)$ & $0.1389270(59)$ & \\
\hline
\end{tabular}


TABLE XI. Transition point for each observable at $N_{t}=8$.

\begin{tabular}{|c|c|c|c|c|c|c|}
\hline$N_{t}$ & $N_{l}$ & $\kappa$ & $\beta_{\mathrm{t}, P}$ & $\beta_{\mathrm{t}, G}$ & $\beta_{\mathrm{t}, L}$ & $\beta_{\mathrm{t}, \Sigma}$ \\
\hline 8 & 12 & 0.14054 & $1.739825(75)$ & $1.739825(75)$ & $1.740175(99)$ & \\
\hline 8 & 16 & 0.14054 & $1.739930(46)$ & $1.739930(46)$ & $1.739972(46)$ & \\
\hline 8 & 12 & 0.14024 & $1.749791(96)$ & $1.749791(96)$ & $1.75033(12)$ & \\
\hline 8 & 16 & 0.14024 & $1.749894(28)$ & $1.749894(28)$ & $1.749997(27)$ & $1.749950(19)$ \\
\hline 8 & 20 & 0.14024 & $1.749906(22)$ & $1.749906(22)$ & $1.749940(22)$ & $1.749909(14)$ \\
\hline 8 & 24 & 0.14024 & $1.749887(19)$ & $1.749887(19)$ & $1.749905(19)$ & $1.749880(22)$ \\
\hline 8 & 12 & 0.13995 & $1.75975(11)$ & $1.75975(11)$ & $1.76047(13)$ & \\
\hline 8 & 16 & 0.13995 & $1.760098(31)$ & $1.760098(31)$ & $1.760305(32)$ & $1.760201(35)$ \\
\hline 8 & 20 & 0.13995 & $1.760185(26)$ & $1.760185(26)$ & $1.760268(27)$ & $1.760230(30)$ \\
\hline 8 & 24 & 0.13995 & $1.760207(35)$ & $1.760207(35)$ & $1.760294(35)$ & $1.760255(25)$ \\
\hline 8 & 12 & 0.13966 & $1.769664(99)$ & $1.769663(99)$ & $1.77086(14)$ & \\
\hline 8 & 16 & 0.13966 & $1.769956(88)$ & $1.769955(88)$ & $1.770396(96)$ & \\
\hline 8 & 20 & 0.13966 & $1.770061(65)$ & $1.770062(65)$ & $1.770267(74)$ & \\
\hline 8 & 12 & 0.1394 & $1.77816(15)$ & $1.77816(15)$ & $1.77995(16)$ & \\
\hline 8 & 16 & 0.1394 & $1.778857(96)$ & $1.778857(96)$ & $1.77956(13)$ & \\
\hline 8 & 20 & 0.1394 & $1.77893(11)$ & $1.77892(12)$ & $1.77933(14)$ & \\
\hline
\end{tabular}

TABLE XII. Value of kurtosis at transition point for each observable at $N_{t}=4,6$.

\begin{tabular}{|c|c|c|c|c|c|c|}
\hline$N_{t}$ & $N_{l}$ & $\beta$ & $K_{\mathrm{t}, P}$ & $K_{\mathrm{t}, G}$ & $K_{\mathrm{t}, L}$ & $K_{\mathrm{t}, \Sigma}$ \\
\hline 4 & 6 & 1.60 & $-1.231(45)$ & $-1.219(46)$ & $-1.103(39)$ & $-1.530(47)$ \\
\hline 4 & 8 & 1.60 & $-1.401(29)$ & $-1.396(28)$ & $-1.303(26)$ & $-1.528(40)$ \\
\hline 4 & 10 & 1.60 & $-1.451(46)$ & $-1.449(48)$ & $-1.386(53)$ & $-1.540(36)$ \\
\hline 4 & 12 & 1.60 & $-1.466(56)$ & $-1.466(55)$ & $-1.434(47)$ & $-1.508(84)$ \\
\hline 4 & 16 & 1.60 & $-1.628(58)$ & $-1.628(57)$ & $-1.609(52)$ & $-1.677(87)$ \\
\hline 4 & 6 & 1.65 & $-0.911(14)$ & $-0.899(14)$ & $-0.877(13)$ & $-1.323(22)$ \\
\hline 4 & 8 & 1.65 & $-0.833(26)$ & $-0.823(26)$ & $-0.792(22)$ & $-1.100(28)$ \\
\hline 4 & 10 & 1.65 & $-0.792(45)$ & $-0.788(45)$ & $-0.755(41)$ & $-0.960(50)$ \\
\hline 4 & 12 & 1.65 & $-0.583(26)$ & $-0.579(25)$ & $-0.569(24)$ & $-0.625(46)$ \\
\hline 4 & 16 & 1.65 & $-0.380(41)$ & $-0.377(41)$ & $-0.354(38)$ & $-0.445(69)$ \\
\hline 4 & 6 & 1.70 & $-0.617(16)$ & $-0.600(16)$ & $-0.677(15)$ & \\
\hline 4 & 8 & 1.70 & $-0.441(29)$ & $-0.432(28)$ & $-0.479(27)$ & \\
\hline 4 & 10 & 1.70 & $-0.333(35)$ & $-0.327(34)$ & $-0.296(32)$ & \\
\hline 6 & 10 & 1.715 & $-1.308(56)$ & $-1.299(56)$ & $-1.243(32)$ & $-1.518(57)$ \\
\hline 6 & 12 & 1.715 & $-1.529(32)$ & $-1.523(32)$ & $-1.446(79)$ & $-1.680(51)$ \\
\hline 6 & 16 & 1.715 & $-1.90(21)$ & $-1.89(21)$ & $-1.79(17)$ & $-1.98(22)$ \\
\hline 6 & 10 & 1.73 & $-1.044(31)$ & $-1.034(31)$ & $-1.004(37)$ & $-1.285(26)$ \\
\hline 6 & 12 & 1.73 & $-1.034(53)$ & $-1.026(53)$ & $-0.997(56)$ & $-1.198(74)$ \\
\hline 6 & 16 & 1.73 & $-1.122(52)$ & $-1.117(52)$ & $-1.108(50)$ & $-1.240(55)$ \\
\hline 6 & 24 & 1.73 & $-1.14(14)$ & $-1.14(14)$ & $-1.08(14)$ & $-1.25(18)$ \\
\hline 6 & 10 & 1.75 & $-0.638(17)$ & $-0.625(17)$ & $-0.737(21)$ & $-1.008(39)$ \\
\hline 6 & 12 & 1.75 & $-0.616(24)$ & $-0.605(24)$ & $-0.698(26)$ & $-0.879(37)$ \\
\hline 6 & 16 & 1.75 & $-0.431(33)$ & $-0.425(33)$ & $-0.479(33)$ & $-0.618(50)$ \\
\hline 6 & 10 & 1.77 & $-0.353(31)$ & $-0.340(30)$ & $-0.639(36)$ & \\
\hline 6 & 12 & 1.77 & $-0.316(38)$ & $-0.308(37)$ & $-0.457(40)$ & \\
\hline 6 & 16 & 1.77 & $-0.226(31)$ & $-0.220(31)$ & $-0.233(42)$ & \\
\hline
\end{tabular}


TABLE XIII. Value of kurtosis at transition point for each observable at $N_{t}=8$.

\begin{tabular}{lccccc}
\hline \hline$N_{t}$ & $N_{l}$ & $\kappa$ & $K_{\mathrm{t}, P}$ & $K_{\mathrm{t}, G}$ & $K_{\mathrm{t}, L}$ \\
\hline 8 & 12 & 0.14054 & $-1.126(32)$ & $-1.118(32)$ & $-0.41(60)$ \\
8 & 16 & 0.14054 & $-1.411(23)$ & $-1.406(23)$ & $-1.17(17)$ \\
8 & 12 & 0.14024 & $-0.856(37)$ & $-0.847(37)$ & $-0.29(48)$ \\
8 & 16 & 0.14024 & $-1.010(25)$ & $-1.003(25)$ & $-0.93(12)$ \\
8 & 20 & 0.14024 & $-1.103(32)$ & $-1.098(32)$ & $-0.923(80)$ \\
8 & 24 & 0.14024 & $-1.126(51)$ & $-1.122(51)$ & $-1.03(10)$ \\
8 & 12 & 0.13995 & $-0.581(38)$ & $-0.570(38)$ & $-0.20(45)$ \\
8 & 16 & 0.13995 & $-0.684(24)$ & $-0.676(24)$ & $-0.74(16)$ \\
8 & 20 & 0.13995 & $-0.648(34)$ & $-0.642(34)$ & $-0.65(17)$ \\
8 & 24 & 0.13995 & $-0.488(64)$ & $-0.483(64)$ & $-0.43(21)$ \\
8 & 12 & 0.13966 & $-0.399(37)$ & $-0.388(37)$ & $-0.57(53)$ \\
8 & 16 & 0.13966 & $-0.389(53)$ & $-0.381(52)$ & $-0.48(41)$ \\
8 & 20 & 0.13966 & $-0.357(57)$ & $-0.351(56)$ & $-0.21(32)$ \\
8 & 12 & 0.1394 & $-0.247(50)$ & $-0.238(50)$ & $-0.26(30)$ \\
8 & 16 & 0.1394 & $-0.240(37)$ & $-0.234(36)$ & $-0.79(30)$ \\
8 & 20 & 0.1394 & $-0.214(47)$ & $-0.206(47)$ & $-0.08(30)$ \\
\hline \hline
\end{tabular}

\section{APPENDIX C: OBSERVABLES}
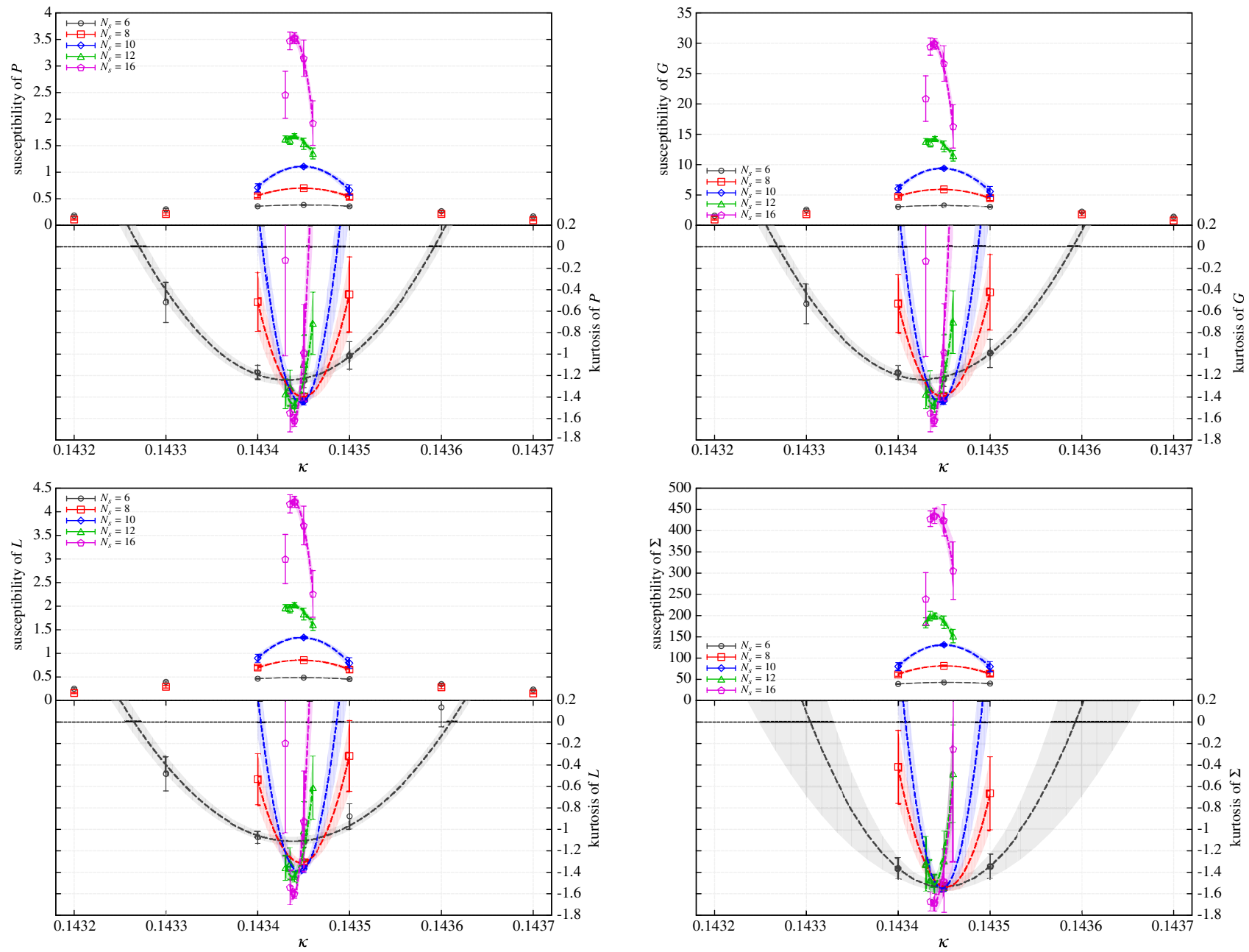

FIG. 9 (color online). Susceptibility and kurtosis as functions of $\kappa$ at $\beta=1.60$ and $N_{t}=4$, together with quadratic fits. Gluon observable $P$ (top-left panel), $G$ (top-right panel), $L$ (bottom-right panel), and quark observable $\Sigma$ (bottom-left panel) are shown. 

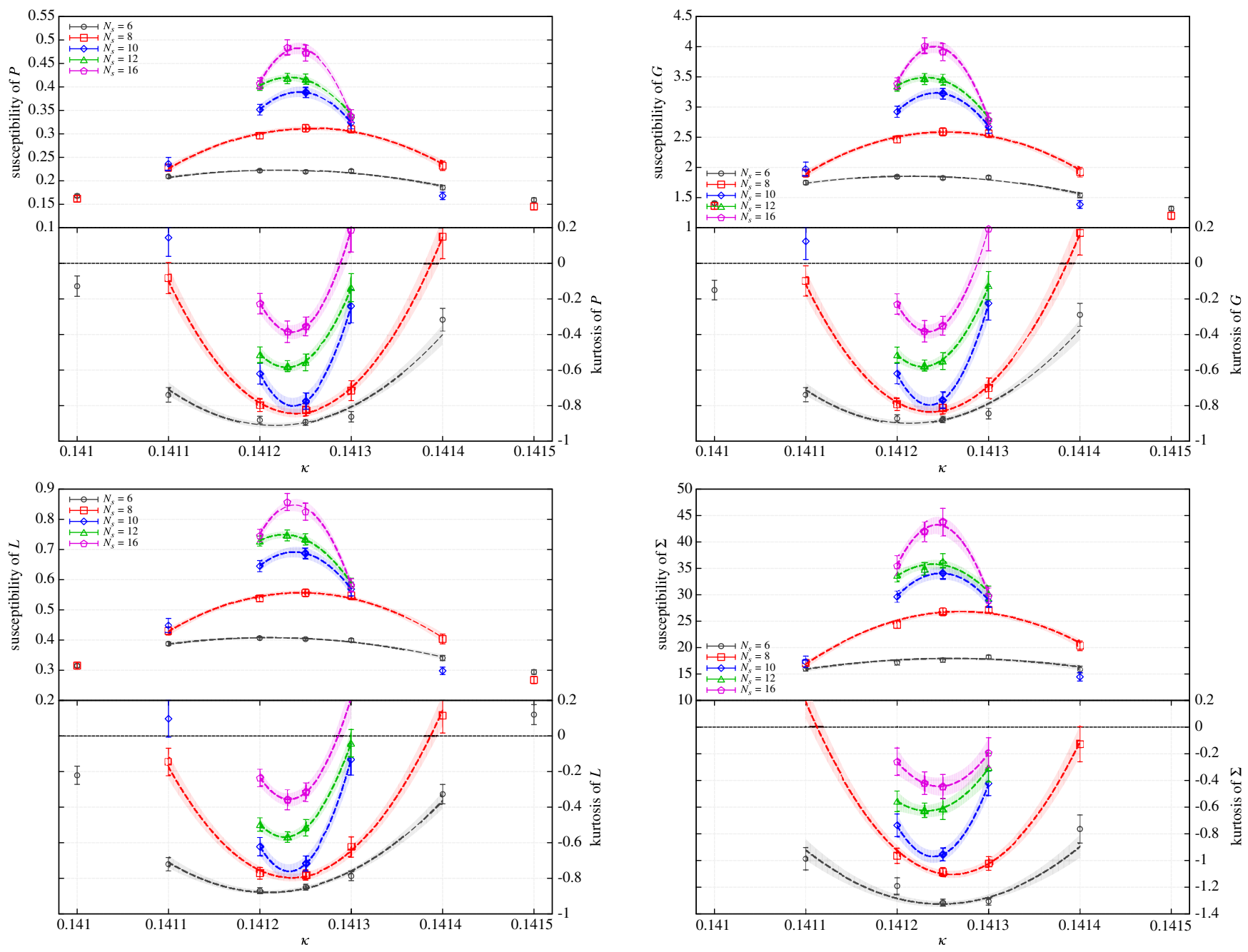

FIG. 10 (color online). Same as Fig. 9, but at $\beta=1.65$ and $N_{t}=4$. 
JIN et al.
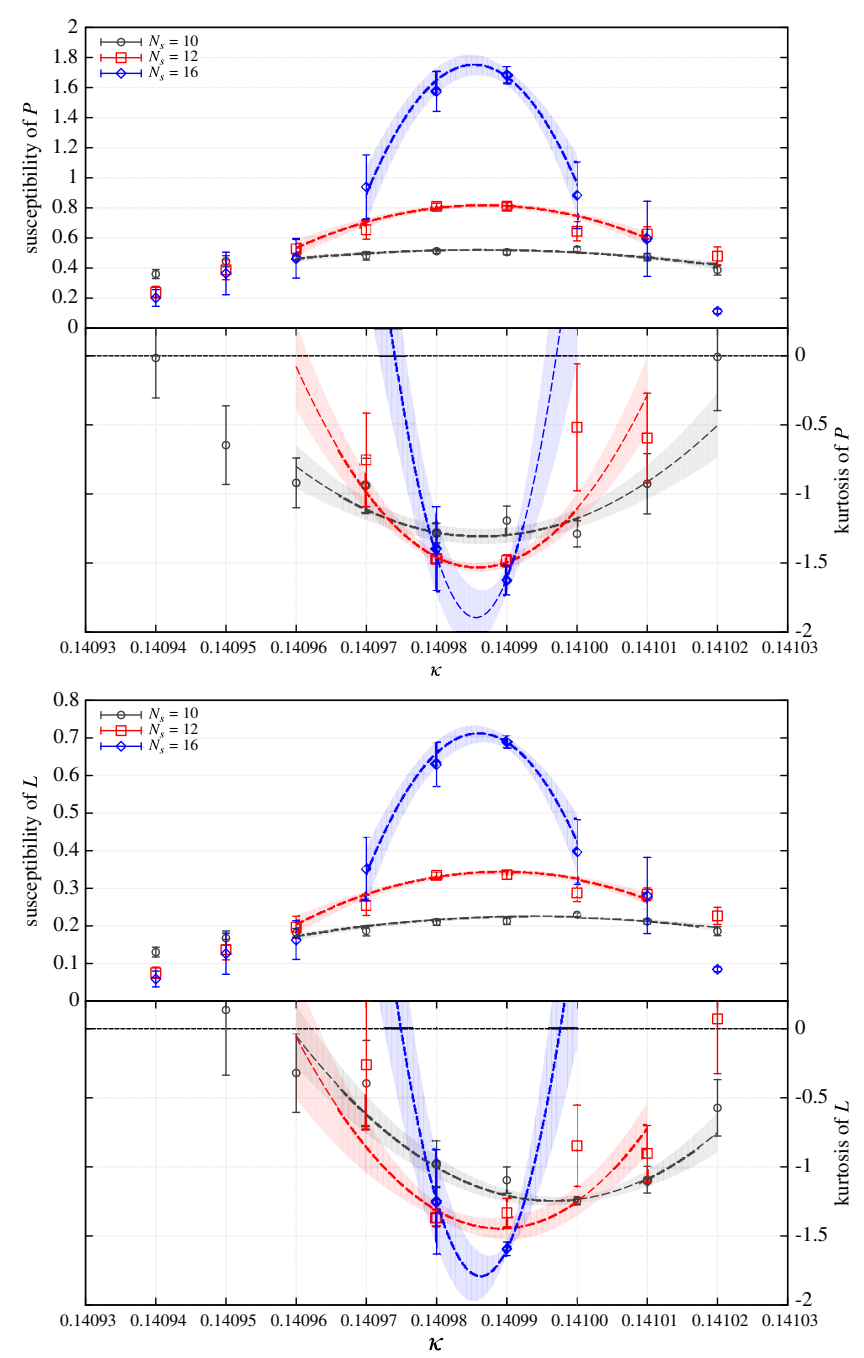

PHYSICAL REVIEW D 91, 014508 (2015)
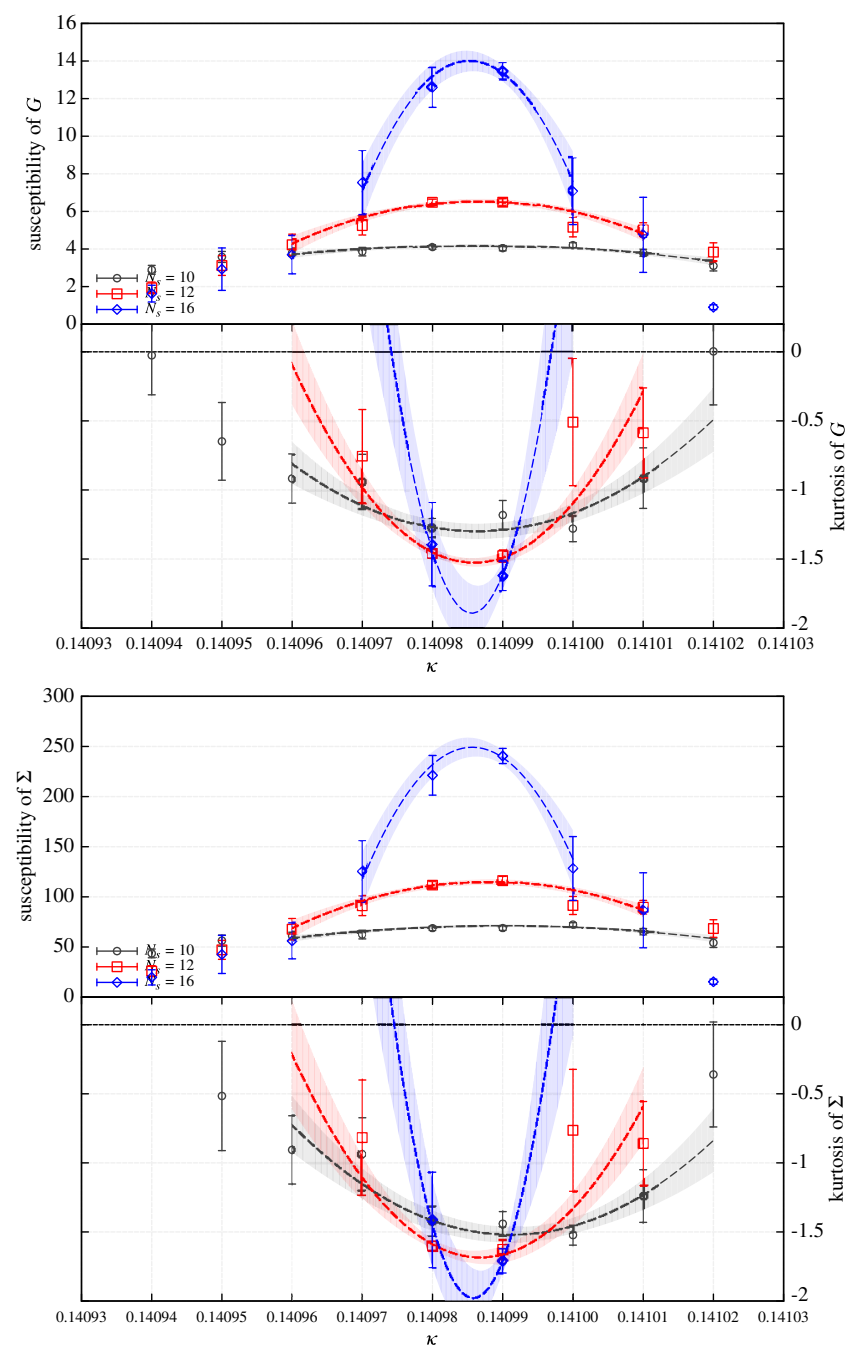

FIG. 11 (color online). Susceptibility and kurtosis as functions of $\kappa$ at $\beta=1.715$ and $N_{t}=6$, together with quadratic fits. Gluon observables $P$ (top-left panel), $G$ (top-right panel), and $L$ (bottom-right panel) and quark observable $\Sigma$ (bottom-left panel) are shown. 

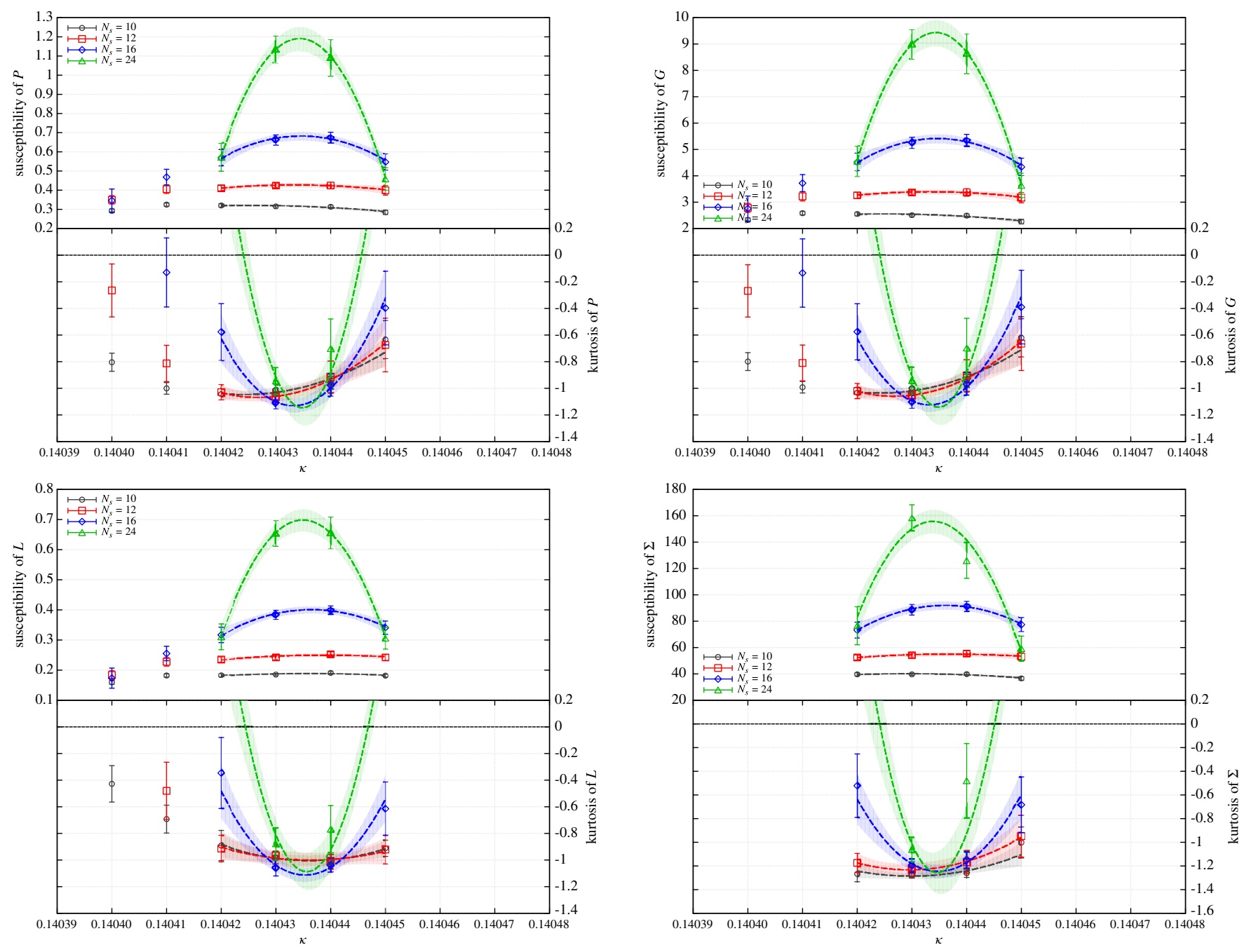

FIG. 12 (color online). Same as Fig. 11 , but at $\beta=1.73$ and $N_{t}=6$. 
JIN et al.
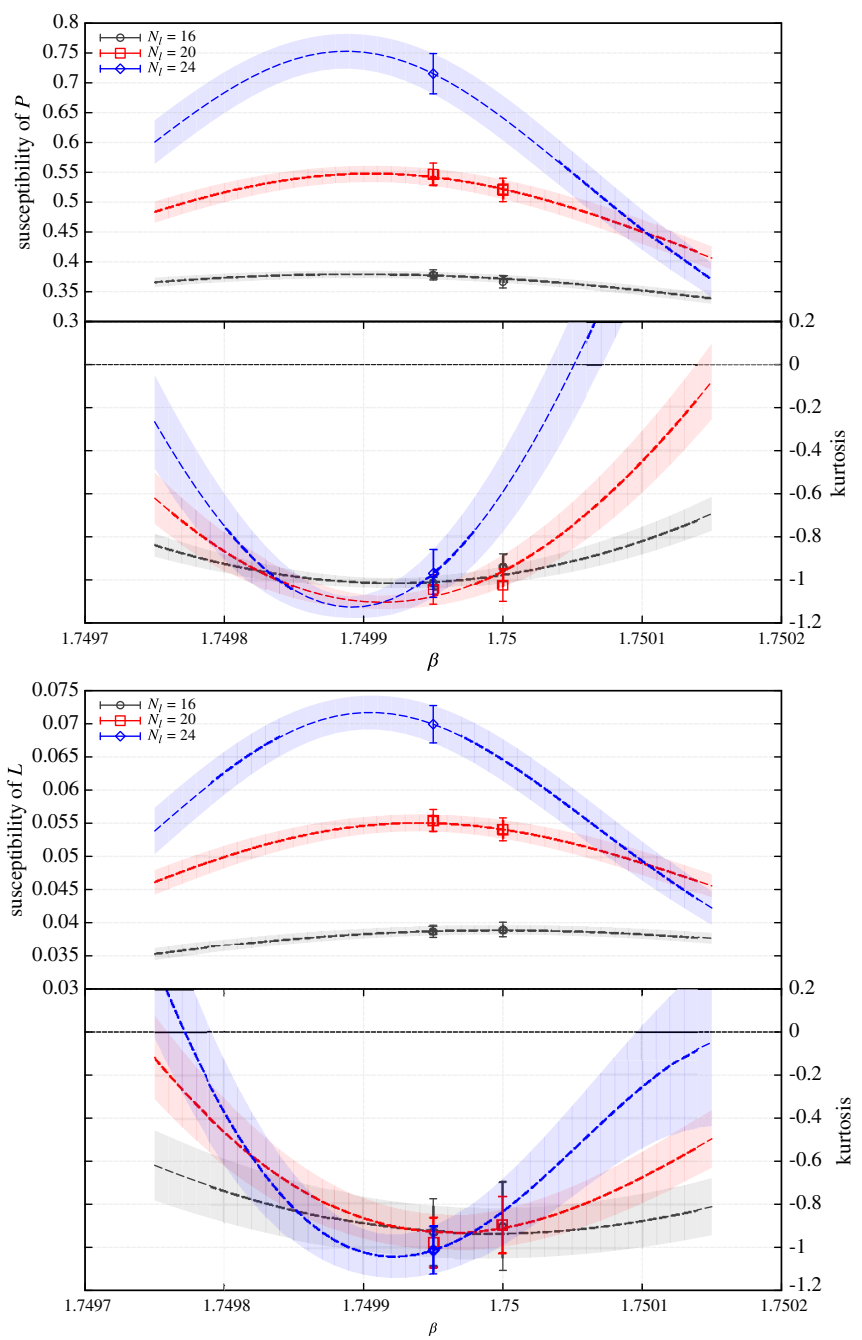

PHYSICAL REVIEW D 91, 014508 (2015)
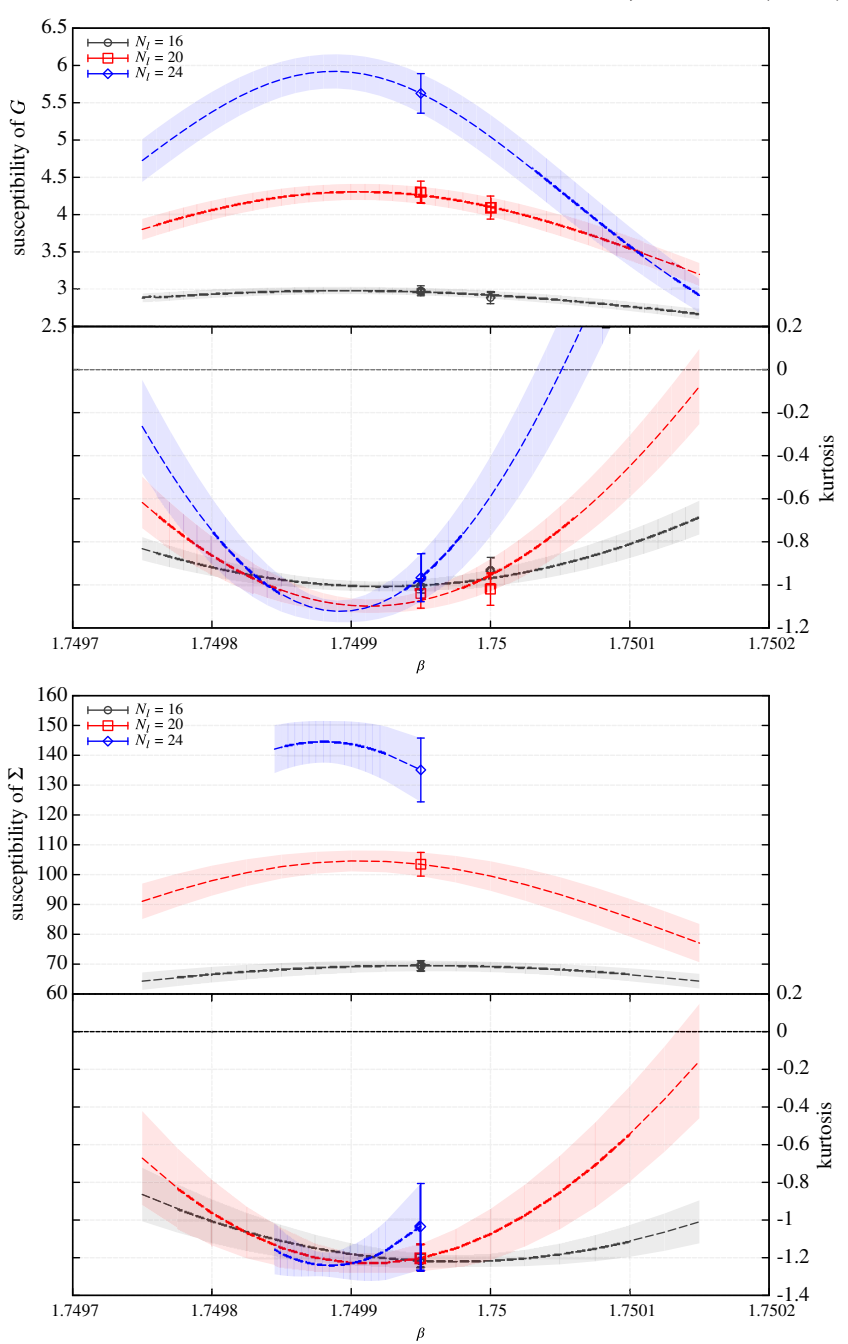

FIG. 13 (color online). Susceptibility and kurtosis as functions of $\beta$ at $\kappa=0.14024$ and $N_{t}=8$, with curves showing reweighted estimates. Gluon observables $P$ (top-left panel), $G$ (top-right panel), and $L$ (bottom-right panel) and quark observable $\Sigma$ (bottom-left panel) are shown. 

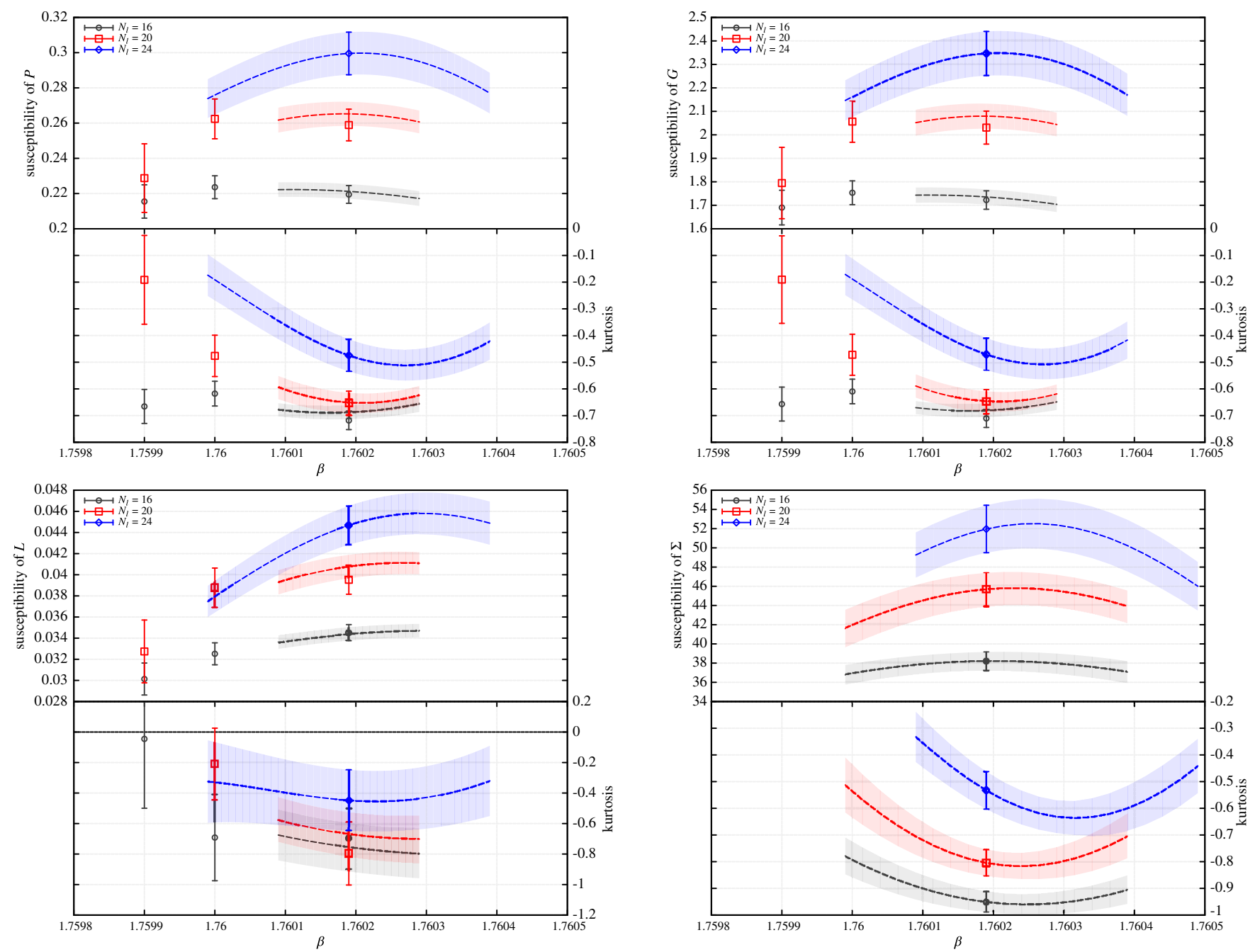

FIG. 14 (color online). Same as Fig. 13, but at $\kappa=0.13995$ and $N_{t}=8$.

[1] R. D. Pisarski and F. Wilczek, Phys. Rev. D 29, 338 (1984).

[2] S. Gavin, A. Gocksch, and R. D. Pisarski, Phys. Rev. D 49, R3079 (1994).

[3] S. Aoki et al. (JLQCD Collaboration), Nucl. Phys. B, Proc. Suppl. 73, 459 (1999).

[4] F. Karsch, E. Laermann, and C. Schmidt, Phys. Lett. B 520, 41 (2001).

[5] P. de Forcrand and O. Philipsen, J. High Energy Phys. 01 (2007) 077.

[6] D. Smith and C. Schmidt, Proc. Sci., LATTICE2011 (2011) 216 [arXiv:1109.6729].

[7] G. Endrődi et al., Proc. Sci., LATTICE2007 (2007) 182 [arXiv:0710.0998].

[8] H.-T. Ding et al., Proc. Sci., LATTICE2011 (2011) 191 [arXiv:1111.0185].

[9] Y. Iwasaki, K. Kanaya, S. Kaya, S. Sakai, and T. Yoshié, Phys. Rev. D 54, 7010 (1996).

[10] A. Bazavov et al., Phys. Rev. D 85, 054503 (2012).

[11] A. Billoire, R. Lacaze, and A. Morel, Nucl. Phys. B370, 773 (1992).
[12] A. M. Ferrenberg and R. H. Swendsen, Phys. Rev. Lett. 61, 2635 (1988).

[13] Y. Iwasaki, Report No. UTHEP-118, 1983.

[14] S. Aoki et al. (CP-PACS and JLQCD Collaborations), Phys. Rev. D 73, 034501 (2006).

[15] Y. Nakamura and H. Stüben, Proc. Sci., LATTICE2010 (2010) 040 [arXiv:1011.0199].

[16] M. Hasenbusch, Phys. Lett. B 519, 177 (2001); M. Hasenbusch and K. Jansen, Nucl. Phys. B659, 299 (2003).

[17] M. A. Clark and A. D. Kennedy, Phys. Rev. Lett. 98, 051601 (2007).

[18] I. P. Omelyan, I. M. Mryglod, and R. Folk, Phys. Rev. E 65, 056706 (2002); Comput. Phys. Commun. 151, 272 (2003).

[19] J. C. Sexton and D. H. Weingarten, Nucl. Phys. B380, 665 (1992).

[20] R. Brower, T. Ivanenko, A. Levi, and K. Orginos, Nucl. Phys. B484, 353 (1997).

[21] M. Lüscher, J. High Energy Phys. 08 (2010) 071.

[22] S. Borsanyi et al., J. High Energy Phys. 09 (2012) 010.

[23] R. Horsley et al., Proc. Sci., LATTICE2013 (2013) 249 [arXiv:1311.5010]. 